\title{
Parametric System Identification of Resonant Micro/Nanosystems Operating in a Nonlinear Response Regime
}

\author{
A.B. Sabater ${ }^{\mathrm{a}}$, J.F. Rhoads ${ }^{\mathrm{a}, *}$ \\ ${ }^{a}$ School of Mechanical Engineering, Birck Nanotechnology Center, and Ray W. Herrick \\ Laboratories, Purdue University, West Lafayette, IN, 47907, USA
}

\begin{abstract}
The parametric system identification of macroscale resonators operating in a nonlinear response regime can be a challenging research problem, but at the micro- and nanoscales, experimental constraints add additional complexities. For example, due to the small and noisy signals micro/nanoresonators produce, a lock-in amplifier is commonly used to characterize the amplitude and phase responses of the systems. While the lock-in enables detection, it also prohibits the use of established time-domain, multi-harmonic, and frequency-domain methods, which rely upon time-domain measurements. As such, the only methods that can be used for parametric system identification are those based on fitting experimental data to an approximate solution, typically derived via perturbation methods and/or Galerkin methods, of a reduced-order model. Thus, one could view the parametric system identification of micro/nanosystem operating in a nonlinear response regime as the amalgamation of four coupled sub-problems: nonparametric system identification, or proper experimental design and data acquisition; the generation of physically-consistent reduced-order models; the calculation of accurate approximate responses; and the application of nonlinear least-squares parameter estimation. This work is focused on the theoretical foundations that underpin each of these sub-problems, as the methods used to address one sub-problem can strongly influence the results of another. To provide
\end{abstract}

\footnotetext{
*Corresponding author

Email addresses: asabater@purdue.edu (A.B. Sabater), jfrhoads@purdue.edu (J.F. Rhoads)
}

Preprint submitted to Mechanical Systems and Signal Processing

November 22, 2015

(C) 2016. This manuscript version is made available under the Elsevier user license http://www.elsevier.com/open-access/userlicense/1.0/ 
context, an electromagnetically-transduced microresonator is used as an example. This example provides a concrete reference for the presented findings and conclusions.

Keywords: Parametric System Identification, Nonlinear, Resonance, Microcantilever, Micro/Nanosystems, Averaging, Perturbation Theory, Harmonic Balance, MEMS, NEMS

\section{Introduction}

There are many established uses of resonant microelectromechanical systems (MEMS) in inertial and pressure sensing applications [1, 2], as well as emerging applications such as mass sensing [3], filtering [4] and timing [5], which are enabled by the small size and low power consumption metrics associated with these systems. As these devices continue to shrink to the nanoscale, the aforementioned advantages are often double-edged in that system characterization becomes more challenging. For example, the dynamic range, or the range of excitations where the response is linear, decreases with a reduction in scale [6], which inhibits the use of established linear characterization techniques.

System identification in the presence of nonlinearity can be a challenging problem. Due to the relevance of this problem to systems of many different scales, it has spawned a vast amount of research literature, of which a review can be found in [7]. Unfortunately, due to experimental constraints, the majority of system identification methods that have been developed cannot be used with micro/nanosystems, as a lock-in amplifier is used to acquire the typically small and noisy response of these systems [8, 9, 10, 11]. This largely inhibits the use of time-domain methods, multi-harmonic methods, or frequency-domain methods, which rely upon a time-domain measurement, as the lock-in amplifier is only capable of measuring the amplitude and phase of a single harmonic of the steady-state response. The methods that are amenable to use when a lock-in amplifier is employed in the measurement system are based on fitting experimental data to an approximate solution of a reduced-order model. These approximate solution-based methods have applicability beyond micro/nanosystem characterization, as they have also been applied in the parametric identification of macroscale systems $[12,13$, 14, 15, 16]. More recent works have adapted approximate solution-based methods for resonant micro/nanosystems $[11,17,18,19,20,21,22]$, but 
there are significant limitations. This work is focused on these limitations, and more generally the conditions under which a resonant micro/nanosystem can be characterized by approximate solution-based methods.

In order to facilitate the parametric system identification of a resonant micro/nanosystem operating in a nonlinear response regime when a lock-in amplifier is employed, several sub-problems must be addressed: nonparametric system identification, or experimental design and data acquisition; the generation of physically-consistent reduced-order models; the calculation of accurate approximate responses; and the application of nonlinear leastsquares parameter estimation methods. This work is focused on the theoretical foundations of these sub-problems, and how each is intrinsically coupled to the others. Accordingly, a general theoretical framework is presented that is independent of the final application of the estimated parameters. Thus, while one could potentially relax constraints that one of the sub-problems might introduce based on the final application, a common framework allows one to communicate with a larger audience, enables technical advancements in one application to benefit all of them, and mitigates the need for subjective expert knowledge.

Since this work is theoretical in nature, the only nonparametric system identification issue considered herein is noise. As will be shown in later sections, with a sufficiently accurate model, noise only influences the variability of the parameter estimates. Accordingly, the bulk of this work is focused on the latter of the three sub-problems noted above. It is important to point out that few prior works have, as identified by the authors, considered all four major aspects of parametrically identifying resonant systems operating in a nonlinear response regime. A notable exception, however, that focused on macroscale systems was [23]. In that work, a multi-term harmonic balance solution was used to produce steady-state responses for a model that included often-ignored, higher-than-third-order nonlinearities. Two major challenges that were also encountered by the authors of this work were issues related to converging to the correct solution, in particular when multiple stable solutions were present, and the sensitivity of the fully-nonlinear estimation technique to initial parameter estimates. The authors of [23] elected to use a sub-optimal cost function as the fully nonlinear estimation technique was sensitive to initial parameter estimates. A significant limitation with using a sub-optimal estimation technique is that it does not necessarily satisfy the requirements needed for statical inference, such as generating confidence intervals for the parameter estimates, performing hypothesis testing to com- 
pare the estimated model to the nominal one or a lower-order version, or ascertaining model adequacy.

While by no means the only way of modeling resonant micro/nanosystems, reduced-order methods are often preferable over others as they can accurately describe dynamic responses in a manner that can be used with the other sub-problems. Without considering the other sub-problems, though, this notion of accuracy can be subjective. There are many ways that the accuracy of a reduced-order model can be improved, such as the inclusion of multiple modes, but in this work, it will be shown that differences between third-order and fifth-order models at excitations close to the critical one, or the one at which bistability is present in the steady-state response, can be significant enough to prohibit accurate parametric identification. A common method of calculating approximate steady-state responses of a micro/nanoresonator is to use first-order perturbation methods. These methods are very useful, particularly in regards to design, as they can distill complex phenomena to something much simpler, but they can also introduce two issues: errors between the first-order solution and higher or more accurate solutions and effective parameter coupling.

There are several ways of estimating system parameters based on observed responses, such as maximum-likelihood methods, but the most commonly used for micro/nanosystem characterization are based on least-squares methods. In these methods, parameter estimation results from minimizing an error function that is equal to the squared sum of the residuals between the model and data. A fundamental assumption is that in the absence of noise, the nominal parameters yield an error function equal to zero. If a first-order perturbation-based solution, or more generally a deficient model, violates this zero error function condition, the estimated parameters may not correspond to the nominal parameters (i.e. the physical significance of the estimated parameters may be dubious). It is important to note that the use of a highfidelity model introduces its own problems, such as the classic bias-variance issue [24]. However, since careful experimental design in conjuction with the proper use of a lock-in amplifier can significantly reduce the variability of the measured response, it is possible to estimate parameters that correspond to a high-fidelity model to within a reasonable tolerance. In addition, in order for the nominal parameters to minimize the error function, the error function's Hessian, evaluated with the nominal parameters, must be positive definite. If the first-order perturbation-based solution effectively couples parameters together, this positive definite condition is violated. Note that this issue of 
effectively coupled parameters is not limited to perturbation-based solutions, and can occur with any solution when its corresponding error function's Hessian is not positive definite.

The starting point for this research related to the parametric system identification of resonators operating in a nonlinear response regime arose from characterizing microscale cantilevers. Accordingly, Section 2 presents a fifth-order model for an electromagnetically-transduced microresonator. The model development is very general, so it could also be extended to the modeling of any thin cantilever subject to strain-dependent damping. In Section 3, a harmonic-balance-based method that can produce accurate responses and facilitate numerical experiments is discussed. The following section then uses this method to show the discrepancies between the third- and fifthorder beam models. The primary result of that section is that for the system under study, whose nominal parameters were selected to match an actual electromagnetically-transduced microresonator, the differences between the third- and fifth-order models are significant for excitations greater than the critical current, or the current at which bistability is present in the steadystate response. As is referenced in Section 3, due to the computational complexity of producing accurate harmonic balance responses and, in turn, the challenge of using this method in a least-squares algorithm, Section 5 presents two approximate solutions for the steady-state response of the microcantilever based on first- and second-order averaging. An example of effectively-coupled parameters is presented in that section, and several others that are fairly common are also discussed. Sections 6 and 7 then present the theoretical foundations of nonlinear least-squares and apply it using approximate solutions based on the methods of averaging and harmonic balance, respectively. It is shown that the first-order averaged solution has a limited range of excitations that are accurately described by this solution. The second-order averaged solution, however, has a relatively-larger range of applicability, but estimates some parameters with a large bias. Compared to the averaging-based methods, the presented harmonic-balance-based methods can be used to estimate parameters over a larger range of excitations, but with the interjection of noise, bias-variance issues, as well as model selection issues, become relevant. 


\section{Model Development}

The structure that provided the inspiration for this work is the electromagnetically-transduced microresonator shown in Fig. 1. This device consists of a silicon microcantilever with two $\mathrm{Au} / \mathrm{Cr}$ wire traces that follow the perimeter of the cantilever. The structure is placed on top of a permanent magnet, such that when a current is supplied to the outer wire trace, a Lorentz force is generated. In turn, as the beam moves, an induced electromotive force (EMF) is generated, which can be used to sense the vibrations of the beam. In deriving a model for this system, it is assumed that the magnetic field near the beam is locally uniform, but can be arbitrarily oriented. In addition, the magnetic field is weak enough such that the induced EMF does not alter the supplied current to the device. Previous works have emphasized the effects of magnetic fields that are parallel to the beam [25], but tangential magnetic fields can be used to parametrically excite the beam $[26,27,28]$ as well. Since this device was tested in a vacuum chamber, in an attempt to reduce viscous damping effects, the amplitude of the current needed to observe responses in a nonlinear response regime was relatively small. The authors of this work have considered the dynamic response of similar devices [11], but in the noted effort, the excitations were limited to near the onset of the nonlinear response regime. In order to observe responses in that regime, significant effort was required to mitigate noise-related issues with the excitation and measurement systems. Thus, a motivation for the study of electromagnetically-transduced microresonators, and more generally micro/nanoresonators, operating in a nonlinear response regime is that it allows one to mitigate these experimental problems [6].

The model developed here is very similar to the ones presented in previous works $[28,31,32,33]$, however, it has been modified to include effects related to nonlinear damping. The need for the model to account for nonlinear damping is based on experimental results, however, since nonlinear damping is not the focus of the present work, the justification for it is relegated to Appendix A. It is assumed that all damping effects are small, viscous damping is insignificant, and that the source of dissipation is straindependent viscoelastic effects. Similar work has been done on modeling thin cantilevers subject to viscoelastic damping [34], but the current work attempts to account for the total nonconservative moment and not just the contribution that directly affects the transverse displacement.

One of the fundamental assumptions of modeling thin beams is that bend- 


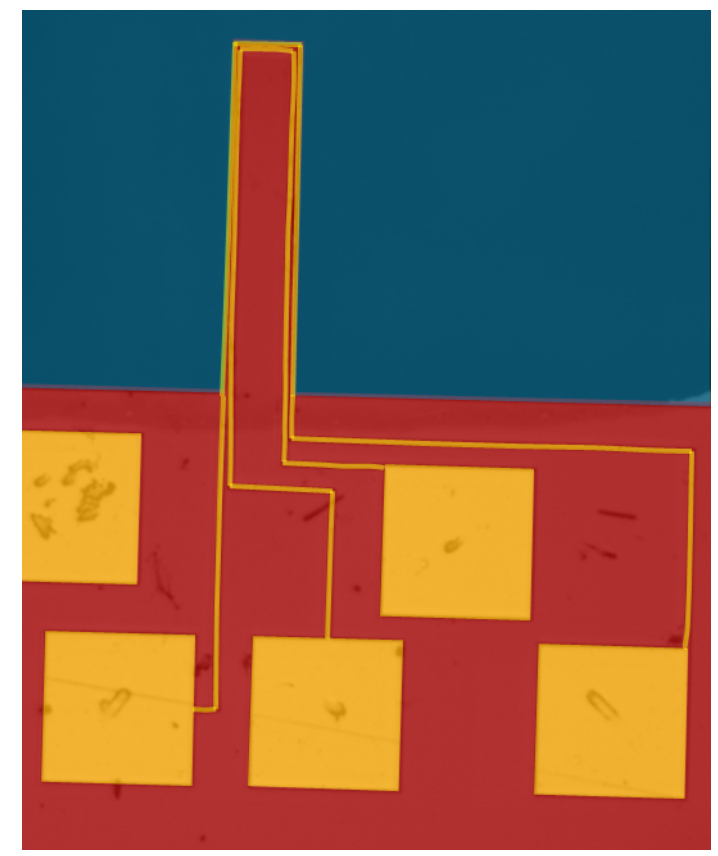

Figure 1: A false-colored micrograph of one of the beams that inspired the presented research. The entire device consists of a cantilever that is nominally $350 \mu \mathrm{m}$ long, $75 \mu \mathrm{m}$ wide, and $2 \mu \mathrm{m}$ thick.

ing is due to moments applied to the beam. Put in a mathematical context,

$$
\epsilon_{s}=-z \psi^{\prime}
$$

where $\epsilon_{s}$ is the axial strain in the beam, $z$ is the distance from the neutral axis of the beam, $\psi$ is defined in the schematic of the beam element in Fig. 2, and $(\bullet)^{\prime}$ denotes a derivative with respect to the arc length variable $s$. Assuming a Kelvin-Voigt material model [35], stress and strain are related by

$$
\sigma_{s}=E \epsilon_{s}+G \dot{\epsilon}_{s}
$$

where $\sigma_{s}$ is the axial stress in the beam, $E$ is the modulus of elasticity, $G$ is the loss modulus, and $(\dot{\bullet})$ denotes a derivative with respect to time. In turn, the bending moment is given by

$$
M=\iint_{A} \sigma_{s} z \mathrm{~d} A=-I\left(E \psi^{\prime}+G \dot{\psi}^{\prime}\right)
$$

where $A$ is the particular cross section under study and $I$ is the cross-sectional moment of inertia. Therefore, the total moment can be said to be due the 
sum of the conservative and nonconservative moments, and it is this nonconservative moment from which nonlinear damping arises. Further details on the selection of the Kelvin-Voigt material model can be found in Appendix A.

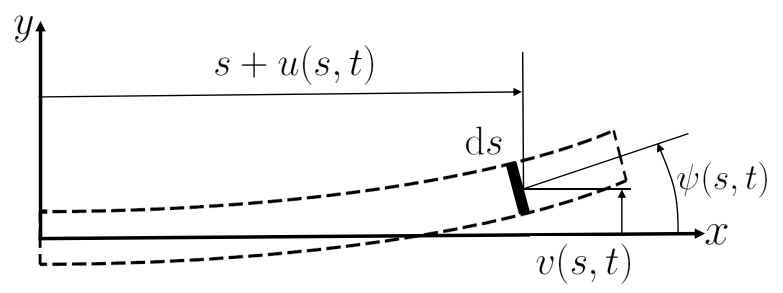

Figure 2: Schematic diagram of the beam element and dynamic variables used for modeling $[28]$.

With Eqn. (3) in hand, a reduced-order model for the dynamics of a thin cantilever beam can be derived using variational principals. To do this, a specific Lagrangian $\bar{L}$ is defined as

$$
\bar{L}=\frac{1}{2} \rho A\left[\dot{u}^{2}+\dot{v}^{2}\right]-\frac{1}{2} E I \psi^{\prime 2},
$$

where $u$ and $v$ are defined in Fig. 2 as the longitudinal and transverse displacements, respectively, and $\rho$ and $A$ are the mass density and cross-sectional area, respectively. It is important to note that this particular Lagrangian prohibits the investigation of certain dynamic phenomena, like torsional vibrations or out-of-plane motion, but previous works have considered flexuralflexural-torsional vibrations $[31,32]$. The assumption of ignoring torsional vibrations is experimentally grounded, as the first bending and torsional modes of the microcantilever shown in Fig. 1 are significantly spaced. These modes were recorded at $22.7 \mathrm{kHz}$ and $209.3 \mathrm{kHz}$, respectively, with a Polytec MSA-400 laser Doppler vibrometer. Since the microcantilever was designed to be weakly sensitive to out-of-plane Lorentz forces and the MSA-400 was not configured to measure motion parallel to the surface it was interrogating, no out-of-plane modes were measured. Effects related to bending due to shear and in-plane torsional inertia that are accounted for in Timoshenko beam models are also neglected as it is assumed that the beam is thin. In addition, the microcantilever shown in Fig. 1 is assumed to be narrow such that plate dynamics can be ignored and the mechanical effects of the two wires along its perimeter are insignificant. These wire traces are assumed to 
be small, but if the device was modified such that the wires were coiled or the beam was much wider, which might be done to decrease the current needed to actuate the beam and increase the induced EMF due to vibrations, the specific Lagrangian may need to be modified [37, 38, 19].

This specific Lagrangian can be used in conjunction with the extended Hamilton's principle to yield the variation equation of motion for the system:

$$
\begin{aligned}
\delta H= & 0 \\
= & \delta \int_{t_{1}}^{t_{2}} \int_{0}^{l}\left\{\bar{L}+\frac{1}{2} \lambda\left[1-\left(1+u^{\prime}\right)^{2}-\left(v^{\prime}\right)^{2}\right]\right\} \mathrm{d} s \mathrm{~d} t \\
& +\int_{t_{1}}^{t_{2}} \int_{0}^{l}\left\{Q_{u} \delta u+Q_{v} \delta v+Q_{\psi^{\prime}} \delta \psi^{\prime}\right\} \mathrm{d} s \mathrm{~d} t,
\end{aligned}
$$

where $l$ is the undeformed length, $\lambda$ is a Lagrange multiplier used to enforce an inextensibility constraint, $Q_{u}$ and $Q_{v}$ are the nonconservative forces in the longitudinal and transverse directions, respectively, and $Q_{\psi^{\prime}}$ is the nonconservative moment applied to the beam. Note that the nonconservative forces and moment are assumed to be zero at the fixed and free ends of the cantilever such that these effects do not need to be accounted for in the boundary conditions. These nonconservative forces and moment, however, must be present to account for both the forces used to actuate the cantilever and effects related to dissipation.

Dissipation in micro/nanosystems is a rather complex and diverse field, as it requires knowledge of many different disciplines [39, 40], but in this effort, as previously noted, it is assumed that all damping effects are strain dependent. Specifically, all damping is assumed to be due to the viscoelastic behavior of the beam's material. The reason for this assumption is that it can account for experimentally-observed nonlinear damping, not only with the system being modeled, but with other nanoresonators operated such that viscous damping is negligible (i.e. operation in vacuum) [41, 21, 22]. Damping due to viscoelastic behavior has been considered before [42], and it is possible other loss mechanisms, such as thermoelastic damping or clamping loss, could also contribute to nonlinear damping mechanisms. The broader goal, however, is to show that any damping effects that are strain dependent will result in a system with nonlinear damping, and that by using the model that will be subsequently presented, an estimate for the influence of nonlinear damping can be ascertained from how strong the linear damping is. That is, in an experimental context, if the excitations are limited such that nonlinear 
effects are small, a quality factor that captures the total contributions of linear damping effects can be extracted. This quality factor can then be used to estimate the loss modulus, provided loss mechanisms are linearly dependent on strain like the previously-discussed model. In turn, this loss modulus can then be used to estimate the influence of nonlinear damping.

It is possible to manipulate Eqn. (5) such that the inclusion of the nonconservative moment alters the nonconservative forces. By integrating the term with $Q_{\psi^{\prime}}$ by parts,

$$
\begin{aligned}
\int_{t_{1}}^{t_{2}} \int_{0}^{l} Q_{\psi^{\prime}} \delta \psi^{\prime} \mathrm{d} s \mathrm{~d} t & =\left.\int_{t_{1}}^{t_{2}} Q_{\psi^{\prime}} \delta \psi \mathrm{d} t\right|_{0} ^{l}-\left.\int_{t_{1}}^{t_{2}}\left\{\left[Q_{\psi^{\prime}}^{\prime} \frac{\partial \psi}{\partial u^{\prime}}\right] \delta u+\left[Q_{\psi^{\prime}}^{\prime} \frac{\partial \psi}{\partial v^{\prime}}\right] \delta v\right\} \mathrm{d} t\right|_{0} ^{l} \\
& +\int_{t_{1}}^{t_{2}} \int_{0}^{l}\left\{\left[Q_{\psi^{\prime}}^{\prime} \frac{\partial \psi}{\partial u^{\prime}}\right]^{\prime} \delta u+\left[Q_{\psi^{\prime}}^{\prime} \frac{\partial \psi}{\partial v^{\prime}}\right]^{\prime} \delta v\right\} \mathrm{d} s \mathrm{~d} t .
\end{aligned}
$$

If ideal cantilever boundary conditions are assumed, the boundary terms vanish, and the end result is that the nonconservative moment effectively adds to the nonconservative forces. From this point forward, the rest of the reduced-order model derivation parallels [28], but a complete derivation follows.

Integrating Eqn. (5) by parts, and assuming that effects related to the nonconservative moment have been included in the nonconservative forces, the variation of the Hamiltonian can be rewritten as

$$
\begin{aligned}
\delta H & =\int_{t_{1}}^{t_{2}} \int_{0}^{l}\left\{-\rho A \ddot{u}+Q_{u}+G_{u^{\prime}}^{\prime}\right\} \delta u \mathrm{~d} s \mathrm{~d} t+\int_{t_{1}}^{t_{2}} \int_{0}^{l}\left\{-\rho A \ddot{v}+Q_{v}+G_{v^{\prime}}^{\prime}\right\} \delta v \mathrm{~d} s \mathrm{~d} t \\
& +\left.\int_{0}^{l} \rho A[\dot{u} \delta u+\dot{v} \delta v] \mathrm{d} s\right|_{t_{1}} ^{t_{2}}+\left.\int_{t_{1}}^{t_{2}}\left\{-G_{u^{\prime}} \delta u-G_{v^{\prime}} \delta v\right\} \mathrm{d} t\right|_{0} ^{l} \\
& +\left.\int_{t_{1}}^{t_{2}}\left[-E I \psi^{\prime}\left(\frac{\partial \psi}{\partial u^{\prime}} \frac{d u^{\prime}}{d v^{\prime}}+\frac{\partial \psi}{\partial v^{\prime}}\right)\right] \delta v^{\prime} \mathrm{d} t\right|_{0} ^{l},
\end{aligned}
$$

where

$$
\begin{aligned}
& G_{u^{\prime}}=-E I \psi^{\prime \prime} \frac{\partial \psi}{\partial u^{\prime}}+\lambda\left(1+u^{\prime}\right), \\
& G_{v^{\prime}}=-E I \psi^{\prime \prime} \frac{\partial \psi}{\partial v^{\prime}}+\lambda v^{\prime} .
\end{aligned}
$$


In order for the variations of $u$ and $v$ to be arbitrary, the terms proportional to their respective variations yield two coupled partial differential equations

$$
\begin{aligned}
& Q_{u}=\rho A \ddot{u}+\left[E I \psi^{\prime \prime} \frac{\partial \psi}{\partial u^{\prime}}-\lambda\left(1+u^{\prime}\right)\right]^{\prime}, \\
& Q_{v}=\rho A \ddot{v}+\left[E I \psi^{\prime \prime} \frac{\partial \psi}{\partial v^{\prime}}-\lambda v^{\prime}\right]^{\prime} .
\end{aligned}
$$

The last three terms of Eqn. (7) yield initial and boundary conditions, which will be assumed to be approximately satisfied. Using the kinematic constraint that there is no bending due to shear forces,

$$
\tan \psi=\frac{v^{\prime}}{1+u^{\prime}}
$$

and the inextensibility constraint,

$$
v^{\prime 2}+\left(1+u^{\prime}\right)^{2}=1
$$

$u$ and $\psi$ can be approximated to fifth-order as

$$
\begin{aligned}
& u^{\prime} \approx-\frac{1}{2} v^{\prime 2}-\frac{1}{8} v^{\prime 4}+O\left(v^{\prime 6}\right), \\
& \psi \approx v^{\prime}+\frac{1}{6} v^{\prime 3}+\frac{3}{40} v^{\prime 5}+O\left(v^{\prime 7}\right) .
\end{aligned}
$$

The results from Eqn. (12) can then be used with the first equation in Eqn. (9) to calculate an approximate Lagrange multiplier

$$
\begin{aligned}
\lambda & \approx-\frac{1}{2} \rho A \int_{l}^{s} \int_{0}^{s_{2}} \ddot{v}^{\prime 2} \mathrm{~d} s_{1} \mathrm{~d} s_{2}-\frac{1}{8} \rho A \int_{l}^{s} \int_{0}^{s_{2}} \ddot{v}^{\prime 4} \mathrm{~d} s_{1} \mathrm{~d} s_{2}-\frac{1}{4} \rho A v^{\prime 2} \int_{l}^{s} \int_{0}^{s_{2}} \ddot{v}^{\prime 2} \mathrm{~d} s_{1} \mathrm{~d} s_{2} \\
& -\left(1+\frac{1}{2} v^{\prime 2}+\frac{3}{8} v^{\prime 4}\right) \int_{l}^{s} Q_{u} \mathrm{~d} s_{1}-E I\left(v^{\prime} v^{\prime \prime \prime}+v^{\prime 2} v^{\prime \prime 2}+v^{\prime 3} v^{\prime \prime \prime}\right) .
\end{aligned}
$$

The approximate Lagrange multiplier can then be used in the second equation of Eqn. (9) to yield a single partial differential equation that describes the 
transverse displacement of the beam

$$
\begin{aligned}
\rho A \ddot{v} & +E I v^{i v}+E I\left[v^{\prime \prime 3}+4 v^{\prime} v^{\prime \prime} v^{\prime \prime \prime}+v^{\prime 2} v^{i v}\right]+E I\left[6 v^{\prime 2} v^{\prime \prime 3}+8 v^{\prime 3} v^{\prime \prime} v^{\prime \prime \prime}+v^{\prime 4} v^{i v}\right] \\
& +\frac{1}{2} \rho A v^{\prime \prime} \int_{l}^{s} \frac{d^{2}}{d t^{2}} \int_{0}^{s_{2}} v^{\prime 2} \mathrm{~d} s_{1} \mathrm{~d} s_{2}+\frac{1}{2} \rho A v^{\prime} \frac{d^{2}}{d t^{2}} \int_{0}^{s} v^{\prime 2} \mathrm{~d} s_{1} \\
& +\frac{1}{8} \rho A v^{\prime \prime} \int_{l}^{s} \frac{d^{2}}{d t^{2}} \int_{0}^{s_{2}} v^{\prime 4} \mathrm{~d} s_{1} \mathrm{~d} s_{2}+\frac{1}{8} \rho A v^{\prime} \frac{d^{2}}{d t^{2}} \int_{0}^{s} v^{\prime 4} \mathrm{~d} s_{1} \\
& +\frac{3}{4} \rho A v^{\prime 2} v^{\prime \prime} \int_{l}^{s} \frac{d^{2}}{d t^{2}} \int_{0}^{s_{2}} v^{\prime 2} \mathrm{~d} s_{1} \mathrm{~d} s_{2}+\frac{1}{4} \rho A v^{\prime 3} \frac{d^{2}}{d t^{2}} \int_{0}^{s} v^{\prime 2} \mathrm{~d} s_{1} \\
& +\left(v^{\prime \prime}+\frac{3}{2} v^{\prime 2} v^{\prime \prime}+\frac{15}{8} v^{\prime 4} v^{\prime \prime}\right) \int_{l}^{s} Q_{u} \mathrm{~d} s_{1}+\left(v^{\prime}+\frac{1}{2} v^{\prime 3}+\frac{3}{8} v^{\prime 5}\right) Q_{u}=Q_{v} .
\end{aligned}
$$

Before proceeding to deriving the reduced-order model, the nonconservative forces can be introduced into the governing equation. For this particular model, the Lorentz force is assumed to only interact with the beam near its free end, but for a more general model, different force profiles can be introduced at this stage. The nonconservative forces on the beam, including viscoelastic effects, are

$$
\begin{aligned}
& Q_{u}=g B_{y} i(t) \delta(s-l)+\left[G I \dot{\psi}^{\prime \prime} v^{\prime}\right]^{\prime}, \\
& Q_{v}=-g B_{x} i(t) \delta(s-l)+\left[-G I \dot{\psi}^{\prime \prime}\left(1+u^{\prime}\right)\right]^{\prime},
\end{aligned}
$$

where $B_{x}$ and $B_{y}$ are the components of the magnetic field in the $x$ and $y$ directions, respectively, $\delta(s)$ is the Dirac delta function and $i(t)$ is the current supplied to the beam. Note that the delta functions are applied an infinitesimal distance from the free end of the beam such that the boundary conditions are not modified. Furthermore, in the schematic of the beam shown in Fig. 2, current is flowing into the page at the tip of the beam.

To help facilitate analysis, Eqn. (14) can be nondimensionalized such that

$$
\hat{v}=\frac{v}{v_{0}}, \quad \hat{s}=\frac{s}{l}, \quad \hat{t}=\frac{t}{T}
$$

where $v_{0}$ is the thickness of the beam and

$$
T=\sqrt{\frac{\rho A l^{4}}{E I}} .
$$


By assuming a single-mode expansion for the dimensionless displacement

$$
\hat{v}(\hat{s}, \hat{t})=z(\hat{t}) \Psi(\hat{s}),
$$

where $\Psi(\hat{s})$ solves the dimensionless boundary value problem for the first bending mode of an ideal cantilever, and rescaling dimensionless time again, a reduced-order model can be derived

$$
\begin{aligned}
z^{\prime \prime} & +\frac{\epsilon}{Q} z^{\prime}+\left[1+\epsilon \lambda_{1} i(\tau)\right] z+\left[\epsilon k_{3}+\epsilon^{2} \lambda_{3} i(\tau)\right] z^{3}+\epsilon \gamma_{3} z^{2} z^{\prime} \\
& +\epsilon \beta\left(z z^{\prime 2}+z^{2} z^{\prime \prime}\right)+\left[\epsilon^{2} k_{5}+\epsilon^{3} \lambda_{5} i(\tau)\right] z^{5}+\epsilon^{2} \gamma_{5} z^{4} z^{\prime} \\
& +\epsilon^{2} \nu_{1} z^{3} z^{\prime 2}+\epsilon^{2} \nu_{2} z^{4} z^{\prime \prime}=\epsilon \eta_{1} i(\tau),
\end{aligned}
$$

where $\epsilon=\left(v_{0} / l\right)^{2}$ and the rest of the parameters are defined in Tables B.6 and B.7. Numerical values for the parameters in Eqn. (19) are given in Table 1. The current supplied to the microcantilever is assumed to be of the form

$$
i(\tau)=i_{0} \cos (\Omega \tau),
$$

where $i_{0}$ is the amplitude of the supplied current and $\Omega$ is the dimensionless ratio of the excitation frequency to the microcantilever's first natural frequency. That is, assuming the excitation frequency is defined as $f_{d}$ in Hertz, then

$$
\Omega=\frac{2 \pi T f_{d}}{\omega_{0}} .
$$

As will be shown in the following section, an approximate solution for Eqn. (19) can be found via the method of averaging. This method, however, requires a small scaling value. The parameter $\epsilon$, which is the square of the beam's aspect ratio, is a natural choice for this parameter, as it allows one to relate a physical quantity, the aspect ratio, to how nonlinear the beam's response is to a given excitation. Assuming that the aspect ratio is small, then Eqn. (19) can be said to describe a weakly-nonlinear resonator [43]. Often this parameter $\epsilon$ is referred to as a "bookkeeping" parameter and is used to denote that certain values in the governing equation are small, but in many efforts that use perturbation-based parametric system identification methods, $\epsilon$ is assumed to be unity. For first-order perturbation methods, this assumption has a small influence on the accuracy of the approximate solution, but with the second-order method that will be derived, assuming $\epsilon=1$ can prohibit parameter estimation. Explicitly, a selection of $\epsilon=1$ can 
yield error functions with rank-deficient approximate Hessians. Beyond prohibiting parameter estimation, it is possible that the interplay between the selection of a too large $\epsilon$ and the approximate form of $\Omega$ can also introduce spurious steady-state responses to second-order solutions that have an $\epsilon$ dependence [44]. The appropriate selection of $\epsilon$ also seems to be an important aspect of modeling micro/nanosystems in general, as this scaling was needed with the harmonic-balance-based identification methods presented in Section 7 .

At this juncture, it is important to note that it is assumed that only a single mode is excited. Since, in general, a Galerkin solution tends to converge to the true solution as the number of modes increases to infinity, this implies that an infinite number of modes are excited. The relative influence of higher-order modes may be small, but as will be shown in the following section, the relative discrepancies between the third- and fifth-order versions of Eqn. (19) can also be small. One of the purposes of this work, however, is to highlight that often neglected effects can complicate or prohibit parametric system identification. Most of this effort focuses on approximate solution and reduced-order model selection, but future works may assess how the inclusion or exclusion of higher-order modes influences parametric system identification of micro/nanosystems operating near their primary mode.

\section{Numerical Experiment Design}

In order to test a given parametric system identification method's capabilities, it is important to have benchmarks or standards. While this assessment could be done with experimental data, this requires the control of well known issues, such as noise, and less well known issues, such as excitation frequency sweep rate and response bias due to the lock-in amplifier's low-pass characteristics. As such, the authors have elected to use numerical experiments for validation within this work. A significant contributing factor for this choice was also the ability to control the underlying model of the system generating the data.

Generation of the data for these numerical experiments requires accurate approximate solutions to Eqn. (19). To this end, the method selected was one similar to the harmonic balance method in [45], except that $\Omega$ was the continuation parameter and a few other modifications were introduced to reduce computational complexity. That is, by rescaling time such that solutions are $2 \pi$ periodic, which aids in simplifying the sampling requirements 
Table 1: Numerical values used in the subsequently-presented numerical experiments as the nominal parameters of Eqn. (19).

$$
\begin{array}{rlrl}
k_{3} & =3.27127 & \lambda_{1} & =0.375962 \frac{g B_{y} l^{4}}{E I v_{0}^{2}} \mathrm{~A}^{-1} \\
k_{5} & =17.3240 & \lambda_{3} & =1.16482 \frac{g B_{y} l^{4}}{E I v_{0}^{2}} \mathrm{~A}^{-1} \\
\beta & =4.59677 & \lambda_{5} & =5.82913 \frac{g B_{y} l^{4}}{E I v_{0}^{2}} \mathrm{~A}^{-1} \\
\nu_{1} & =51.2607 & Q & =0.284413 \frac{E T v_{0}^{2}}{G l^{2}} \\
\nu_{2} & =25.6304 & \gamma_{3} & =17.2528 \frac{G}{E T} \\
\eta_{1}=-0.161781 \frac{g B_{x} l^{5}}{E I v_{0}^{3}} \mathrm{~A}^{-1} & \gamma_{5} & =162.430 \frac{G}{E T}
\end{array}
$$

that are subsequently discussed, and assuming that even harmonics do not contribute to the solution (which is not only expected, as Eqn. (19) does not possess any even nonlinearities, but was also validated with a different set of numerical experiments), the solution to Eqn. (19) was assumed to be of the form

$$
x(\tau)=\sum_{n=1}^{N}\left\{C_{n} \cos ([2 n-1] \tau)+S_{n} \sin ([2 n-1] \tau)\right\} .
$$

Provided $N$ is large enough, the relative error of this method is small. Since the application of this model is for micro/nanosystems sensed using a lock-in detection method, only the first-harmonic of the steady-state response is of interest, thus the steady-state amplitude response is defined as

$$
a=\sqrt{C_{1}^{2}+S_{1}^{2}}
$$

A detailed explanation of how to find the values of $C_{n}$ and $S_{n}$ that satisfy Eqn. (19) can be found in [45], but a succinct explanation follows. Note that the foundations of the method are described in these references [46, 47, 48]. First, Eqn. (19) is modified such that it is equal to zero. Then, for a given 
set of $C_{n}$ and $S_{n}$, a sampled version of this equation is generated. Next, a discrete Fourier transform is performed to estimate the Fourier coefficients of the sampled equation. A solution is found when these $2 N$ coefficients are all equal to zero, so the process of selecting a set of $C_{n}$ and $S_{n}$ is repeated until a solution converges. An advantage of this discrete Fourier transform based method is the ease of increasing or decreasing the number of harmonics in a given approximate solution. A limitation of this method is that it does not allow one to analytically calculate the Jacobian of the system of equations one solves to get a given solution, which is important in a parametric identification method, as this Jacobian is used, in part, to calculate solution sensitivities.

One of the motivating reasons for using continuation methods is that, near turning points, the Jacobian of the system of equations for the $C_{n}$ and $S_{n}$ becomes singular, thus an additional equation is introduced such that the Jacobian is no longer singular. For the harmonic balance method implemented for this work, this equation was of the form

$$
\Delta s^{2}=\left(\Omega-\Omega_{0}\right)^{2}+\frac{2 N}{\sigma_{\text {scale }}^{2}} \sum_{n=1}^{N}\left[\left(C_{n}-C_{n 0}\right)^{2}+\left(S_{n}-S_{n 0}\right)^{2}\right],
$$

where the subscript 0 denotes the previously found solution and the scaling factor $\sigma_{\text {scale }}$, which is needed to aid the convergence of the method, is defined as

$$
\sigma_{\text {scale }}=\frac{1}{\Omega} \sqrt{\sum_{n=1}^{N} C_{n}^{2}+S_{n}^{2}}
$$

To determine the stability of a given solution, Floquet theory was used. Classically, as was done in [45], this amounts to determining the eigenvalues of the monodromy matrix, or characteristic multipliers. If these characteristic multipliers all have a magnitude less then one, then the system is stable, and if this is not true, then the solution is unstable. However, calculating the monodromy matrix via numerical integration can take as long or longer than calculating its associated harmonic balance solution. Instead, a harmonic-based method for computing stability that is founded on computing the eigenvalues of the associated Hill matrix problem is used [49].

In the numerical experiments, $\Delta s=0.02, N=10$ and stable and unstable solutions are denoted by solid and dashed lines, respectively. The justification for the large $N$ used is that accuracy is paramount. For the largest excitation 
considered, the $C_{9}, S_{9}, C_{10}$ and $S_{10}$ terms were on the order of $10^{-16}$ or less. The closeness of the $9^{\text {th }}$ and $10^{\text {th }}$ terms suggests that the $C_{n}$ and $S_{n}$ for $N=10$ are as accurate as can possibly be obtained by the current method and are limited by numerical issues. More generally, references [46, $47,48]$ argue that if certain conditions are met, then the harmonic balance solution uniformly converges to the exact solution for sufficiently high $N$. The first condition is that the solution must be isolated, or has non-unitary characteristic multipliers. The second condition is that if the differential equation can be written as a series of first-order differential equations of the form $\dot{x}=X(x, t)$, the function $X$ must have first-order derivatives with respect to both time and the spatial variables. The criteria of a sufficiently high $N$ may seem nebulous, but these references also provide methods of estimating the error of the harmonic balance solution for a finite value of $N$. Using Stoke's error estimate [48], the error of the harmonic balance solution is less than twice the product of the residual of the harmonic balance solution and a term that is referred to as $M$. Definitions of the residual and this $M$ that are useful for calculation can be found in [47], but as noted by Stokes, for a sufficiently high number of harmonics in the harmonic balance solution, this $M$ converges to a finite value. Thus, the term that dominates the error of the harmonic balance solution is its residual. For clarity and for the specific system being studied, the residual is defined as a constant that must be greater than the maximum difference between the left- and right-hand sides of Eqn. (19). The subtle point to this is that both the previously-referenced terms vary with time.

Figure 3 shows the residual of the harmonic balance solution as a function of the parameter $N$, as defined in this work, for the largest excitation case considered in this work, and with the nominal parameters. For $N=8$ and larger, this residual tends to reach a very small minimum value. As will be shown in a later section, in cases where the residuals between the harmonic balance solution and ones based on averaging are much greater than the one corresponding to the $N=10$ harmonic balance solution, the authors of this work argue that these differences are due to inaccuracies of the averaged solutions. One would be correct to point out that an $N=10$ harmonic balance solution is not computationally efficient, and that the convergence of the residual to a small finite value implies that the accuracy of the harmonic balance solution is limited by its current implementation, but the selection of $N=10$ was guided solely on the need for a benchmark solution where further increases in $N$ would yield no further increases in accuracy. 


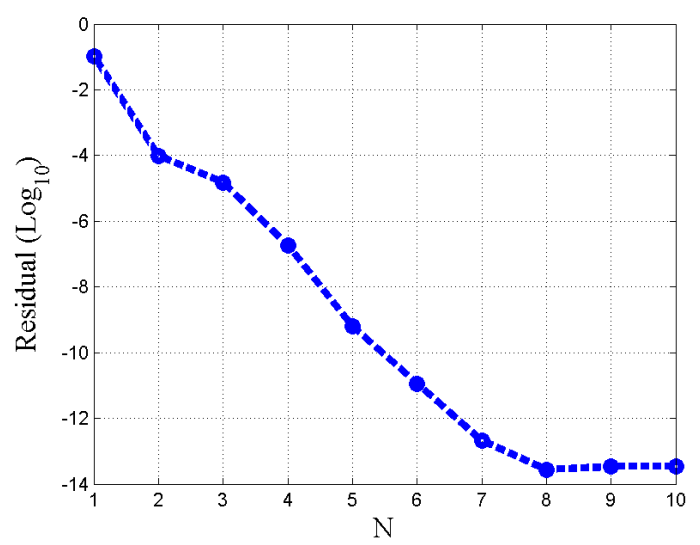

Figure 3: A plot of the residual of the harmonic balance solution as a function of $N$, or the number of harmonics used in the approximate solution, for the largest excitation case considered in this work.

While the previously-described method allows one to generate steadystate responses, typically in an experimental context, one measures the response at discrete frequencies. In addition, these discrete frequencies are selected and changed based on a need to reveal different hysteretic behavior. Therefore, the previously described harmonic balance method needs to be adapted such that steady-state responses at defined frequencies can be calculated, and in a fashion consistent with the previous state of the microresonator. The first step in generating this data is reformulating the response using an amplitude and phase representation, as was done in [45]. While the sine and cosine basis in this work mitigates the issue of multiple equivalent phases, the amplitude and phase representation allows the components of the response to be a smooth functions of $\Omega$. This smoothness is important, as it allows one to interpolate the response at specified frequencies. Next, the amplitude and phase representation of the harmonic balance solution was sampled using a linear interpolation, and in a fashion consistent with slow and discrete forward and reverse sweeps of the excitation frequency. The dimensionless frequency bandwidth considered was from $\Omega=0.9995$ to $\Omega=1.001$ with a $\Delta \Omega$, or the change in $\Omega$ between discrete frequencies, set to be $2 \times 10^{-5}$. For the nominal system discussed in the following sections, this corresponds to the excitation frequency being discretely swept from approximately $11 \mathrm{~Hz}$ below the natural frequency of the microcantilever to approximately $22 \mathrm{~Hz}$ above the natural frequency of the microcantilever in 
steps of $0.4 \mathrm{~Hz}$. Thus, the selection of $\Delta s=0.02$ provided a minimum of four responses between frequencies at which the response was interpolated at, which enabled relatively smooth interpolations. Finally, in order to alleviate interpolation errors, the interpolated solution was then converted back into the sine and cosine basis, and was used as a starting guess for the harmonic balance solution at the specified frequency. This last solution was the one used to generate data and was found to be of nontrivial importance.

\section{Comparison of Third-Order Versus Fifth-Order Beam Models}

Many of the classic works on the nonlinear dynamics of cantilever beams only consider third-order nonlinearities [31, 32, 33]. Third-order models are quite useful, as with minimal adjustments to the linear theory, experimentallyobserved phenomena, such as the hardening or softening nature of the steadystate response or bistability, can be qualitatively predicted. It is assumed, a priori, that third-order models are quantitatively accurate, but there are cases when higher-order models are needed to even be qualitatively accurate [28]. Motivated by such findings, this section explores the differences between the two models, as well as if and when these differences are significant.

Figures 4-6 depict the steady-state amplitude response for $i_{0}=0.4,0.7$ and $1.0 \mathrm{~mA}$, respectively. These three excitations will be subsequently referred to as Cases 1 to 3, respectively. The nominal parameters used to generate these responses, and the ones used in the following sections for system identification, are $\epsilon=(2 / 350)^{2}, Q / \epsilon=6500, \epsilon \eta_{1}=2 \mathrm{~A}^{-1}$ and $B_{y}=$ $0 \mathrm{~T}$. Assuming that the modulus of elasticity $E$ is $159 \mathrm{GPa}$, and the density of silicon is $2330 \mathrm{~kg} / \mathrm{m}^{3}$, then for the dimensions of the beam given in Fig. 1 and the value of $\epsilon \eta_{1}$ given, the in-plane magnetic field $B_{x}$ is approximately $-61 \mathrm{mT}$. The third- and fifth-order models are denoted by red and blue curves, respectively. For Case 1, shown in Fig. 4 and an example of an excitation before bistability is present in the response, there are relatively insignificant differences between the two models. Based on previous works [11], using first-order averaging, the presented scaling, and ignoring nonlinear damping, the critical current, or the current at which bistability is observed in the steady-state response, is

$$
\begin{aligned}
i_{c r} & =\frac{4 \sqrt{2}}{3^{3 / 4}\left(\frac{Q}{\epsilon}\right)^{3 / 2} \sqrt{\left|\epsilon \alpha_{3}\right|}\left|\epsilon \eta_{1}\right|} \\
& =0.53 \mathrm{~mA} .
\end{aligned}
$$


Thus for currents less than this value, it is expected that the two models will agree.

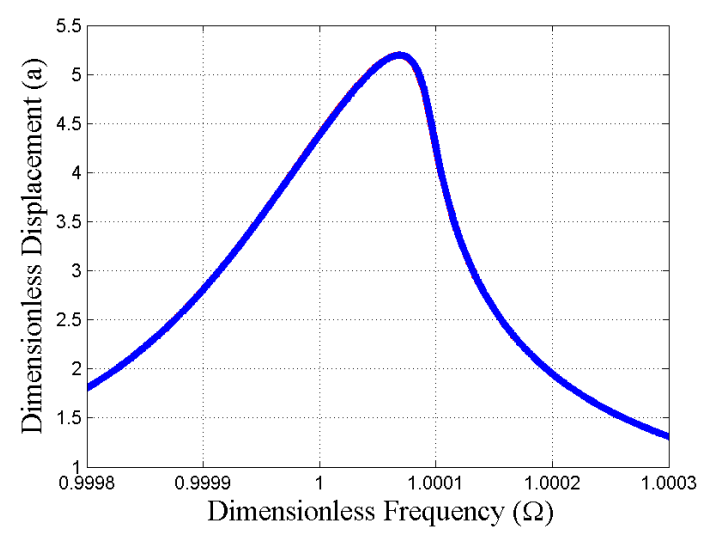

Figure 4: The steady-state responses for Case 1 where $i_{0}$ is $0.4 \mathrm{~mA}$. The third- and fifth-order models are shown in red and blue, respectively.

For currents greater than the critical current, as in Case 2 shown in Fig. 5, there are some differences between the two models. While the error may seem to be insignificant, the difference is great enough to introduce issues with the parameter estimation method presented in the following section. As one might expect, as the excitation increases, the differences between the thirdand fifth-order models increase. This is shown in Fig. 6, which is for Case 3.

Since there is a need for accurate models of nonlinear resonators that can be used for parametric system identification, a balance between computational complexity and implementation must be found. As Figs. 4 to 6 show, third-order models may not sufficiently describe experimental results beyond the critical excitation. Ignoring terms related to parametric excitation, the third- and fifth-order models require five and nine parameters, respectively. Given that nonlinear system identification with five parameters can be challenging, nine parameters can be prohibitively difficult. Thus, in the following section, an approximate solution to the third-order model is found, but it is assumed from the onset that it may fail to accurately model results for currents beyond the critical one. Part of Section 7, in contrast, considers a method to estimate all nine parameters of the fifth-order model. 


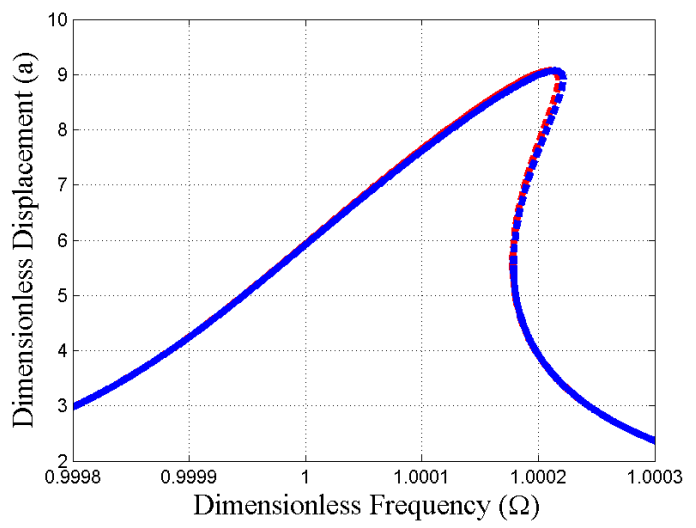

Figure 5: The steady-state responses for Case 2 where $i_{0}$ is $0.7 \mathrm{~mA}$. As in the previous figure, third- and fifth-order models are shown in red and blue, respectively. In addition, stable and unstable solutions are denoted by solid and dashed lines, respectively.

\section{Approximate Solutions Using the Method of Averaging}

While several methods could be used to find an approximate solution to Eqn. (19), the method of averaging was selected because of the accuracy and efficiency of generating an approximate solution. In addition, since the steady-state amplitude predicted by the method of averaging is found by solving a polynomial equation, the implicit function theorem can be used to find solution sensitivities, which in turn can be used in parameter estimation algorithms. Moreover, compared to the method described in Section 3 , calculating stability and generating forward and reverse frequency sweep responses is much more straightforward. Before using this method, though, a coordinate transformation needs to be applied to Eqn. (19) such that the transformed system can be written as a polynomial in $\epsilon$. Specifically, the following constrained coordinate transformation is assumed

$$
\begin{aligned}
z(\tau) & =a(\tau) \cos [\Omega \tau+\phi(\tau)], \\
z^{\prime}(\tau) & =-a(\tau) \Omega \sin [\Omega \tau+\phi(\tau)] .
\end{aligned}
$$

In conjunction with the transformation in Eqn. (27), a constraint is needed such that one can solve for $a(\tau)$ and $\phi(\tau)$

$$
0=a^{\prime} \cos (\Omega \tau+\phi)-a \phi^{\prime} \sin (\Omega \tau+\phi) .
$$

If Eqn. (19) is written in the form of a weakly-nonlinear resonator

$$
z^{\prime \prime}+z=f\left(z, z^{\prime}, t, \epsilon\right),
$$




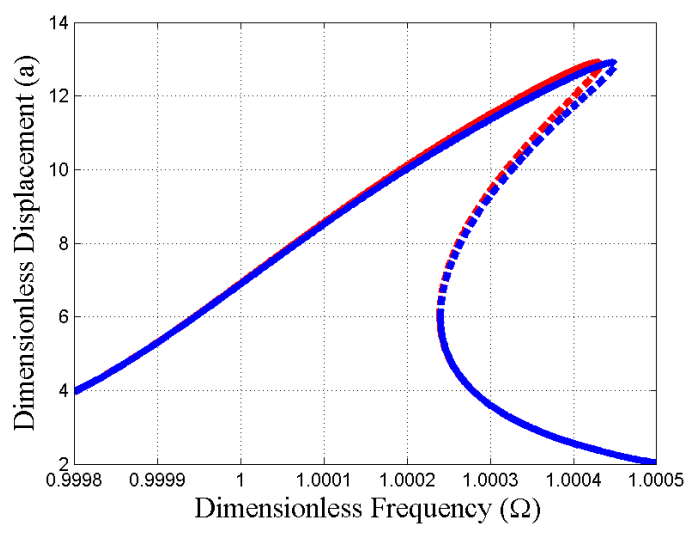

Figure 6: For Case 3, an excitation of $1.0 \mathrm{~mA}$ was considered.

where $f\left(z, z^{\prime}, t, 0\right)=0$, then the transformed version of Eqn. (19) is

$$
\begin{aligned}
& a^{\prime}=-\frac{1}{\Omega}\left[f+\left(\Omega^{2}-1\right) a \cos (\Omega \tau+\phi)\right] \sin (\Omega \tau+\phi), \\
& \phi^{\prime}=-\frac{1}{\Omega a}\left[f+\left(\Omega^{2}-1\right) a \cos (\Omega \tau+\phi)\right] \cos (\Omega \tau+\phi) .
\end{aligned}
$$

At this junction, no approximations of Eqn. (19) have been made. While ideally one might want to develop a system identification method that could uniquely and accurately determine all of the parameters in Eqn. (19), the difficulty of system identification increases with the number of parameters. A much less computationally challenging problem, however, would be the determination of all of the $O(\epsilon)$ parameters, except for $\lambda_{1}$ which is related to parametric excitation. This is akin to ignoring fifth-order nonlinearities, which as shown in the previous section, are relevant for excitations greater than the critical excitation. Thus, provided the excitations are limited, but possibly beyond the critical current, an approximate solution to Eqn. (19) that only considers third-order nonlinearities, and based on second-order averaging [50], could be used to build qualitatively accurate models that have unique parameters. While Section 7 considers the use of a harmonic-balancebased methods, these methods are much more computationally demanding and are sensitive to initial parameter estimates.

In order to derive an approximate solution for Eqn. (19), some additional assumptions are needed. Specifically, the dimensionless excitation frequency 
is redefined as

$$
\Omega=1+\epsilon \sigma,
$$

and to second order the transformed variables are defined to be

$$
\begin{aligned}
& a=a_{0}+\epsilon a_{1}+\epsilon^{2} a_{2}, \\
& \phi=\phi_{0}+\epsilon \phi_{1}+\epsilon^{2} \phi_{2},
\end{aligned}
$$

where $a_{0}$ and $\phi_{0}$ are solutions to the averaged equations

$$
\begin{aligned}
& a_{0}^{\prime}=\epsilon g_{1 a}+\epsilon^{2} g_{2 a}, \\
& \phi_{0}^{\prime}=\epsilon g_{1 \phi}+\epsilon^{2} g_{2 \phi} .
\end{aligned}
$$

The solutions to the averaged equations are of primary interest, as it is assumed that provided $\epsilon$ is sufficiently small, the higher-order contributions to the solution (i.e. $a_{1}$ ) can be ignored. Thus, the averaged equations are

$$
\begin{aligned}
& g_{1 a}=-\frac{4 a+a^{3} Q \gamma_{3}+4 i_{0} Q \eta_{1} \sin (\phi)}{8 Q}, \\
& g_{1 \phi}=\frac{a^{3}\left(3 k_{3}-2 \beta\right)-8 a \sigma-4 i_{0} \eta_{1} \cos (\phi)}{8 a},
\end{aligned}
$$

and

$$
\begin{aligned}
g_{2 a} & =\frac{1}{32 Q}\left(a^{5} k_{3} Q \gamma_{3}+2 a^{5} Q \beta \gamma_{3}-4 a^{3} Q \gamma_{3} \sigma+8 a^{3} \beta+3 a^{2} i_{0} k_{3} Q \eta_{1} \sin \phi\right. \\
& \left.+6 a^{2} i_{0} Q \beta \eta_{1} \sin \phi-a^{2} i_{0} Q \gamma_{3} \eta_{1} \cos \phi+8 i_{0} Q \eta_{1} \sigma \sin \phi+4 i_{0} \eta_{1} \cos \phi\right) \\
g_{2 \phi} & =\frac{1}{256 a Q^{2}}\left(12 a^{5} k_{3} Q^{2} \beta-51 a^{5} k_{3}^{2} Q^{2}+20 a^{5} Q^{2} \beta^{2}-11 a^{5} Q^{2} \gamma_{3}^{2}+96 a^{3} k_{3} Q^{2} \sigma\right. \\
& -64 a^{3} Q^{2} \beta \sigma-48 a^{3} Q \gamma_{3}+72 a^{2} i_{0} k_{3} Q^{2} \eta_{1} \cos \phi+16 a^{2} i_{0} Q^{2} \beta \eta_{1} \cos \phi \\
& \left.-40 a^{2} i_{0} Q^{2} \gamma_{3} \eta_{1} \sin \phi-32 a+64 i_{0} Q^{2} \eta_{1} \sigma \cos \phi-32 i_{0} Q \eta_{1} \sin \phi\right) .
\end{aligned}
$$

Careful analysis of Eqn. (34) will show that with the first-order averaged solution, a set of $k_{3}$ and $\beta$ exist that produce the same response, but this is not the case with the second-order averaged solution. Specifically, for the first-order averaged equations, a parameter

$$
\alpha_{3}=3 k_{3}-2 \beta,
$$


can be defined and will be subsequently referred to as the effective nonlinear coefficient.

If this effective nonlinear coefficient or a similar one is not used when the first-order averaged solution is employed in a least-squares fitting method, it can introduce significant issues. One way of decoupling the geometric and inertial effects would be to also conduct static tests, but this method is not always feasible with micro/nanoresonators. In a more general sense, similar effective nonlinear coefficients have been derived from the models of other micro/nanoresonators, like electromagnetically-actuated microcantilevers with parametric excitation [28] and resonant microbeams with electrostatic excitation [51]. Other effectively-coupled parameters can result from transduction mechanisms, or the way by which input signals produce forces and in turn how displacements produce output signals. This was noted in a previous work by the authors [11], but it is a common issue with micro/nanoresonators that use different phenomena for actuation and sensing. For example, previous works have shown the limitations of capacitive detection of micro/nanoresonators. To combat high feedthrough currents and impedance mismatch, piezoresisitive detection is used [52]. The models for these devices require knowledge about both the piezoresistive gauge factor and the modulus of elasticity. Based on the characterization methods used, these parameters could not be independently determined. Thus, this issue of parameters being effectively coupled by system identification methods is by no means an isolated problem.

\section{Approximate Solution Selection: Fitting to Model-Generated Data with Averaged Solutions}

In order to demonstrate the advantages of the second-order averaged solution over the first-order averaged solution, both of these solutions were used to produce fits to data generated from the previously-presented harmonic balance method in a least-squares sense. Before results can be shown, however, some background information is requisite. An estimate of the parameters for a given model are defined as the parameters that minimize the error function

$$
S(\hat{\mathbf{p}})=\mathbf{R}(\hat{\mathbf{p}})^{T} \mathbf{R}(\hat{\mathbf{p}}),
$$

where $\hat{\mathbf{p}}$ is a $p \times 1$ vector of the parameter estimates, $p$ is the number of parameters for the corresponding model, $\mathbf{R}$ is the $R \times 1$ residual column 
vector between the model and the data and $R$ is the number of measurements. In general, an explicit solution for the minimum of Eqn. (37) cannot be found, so iterative methods are used. While many methods exist to solve this problem [53], the method used here exploits the implicit function theorem and an approximate Hessian. A Taylor series expansion of Eqn. (37) in the neighborhood of $\mathbf{p}^{*}$, assuming that $\mathbf{p}^{*}$ is close to $\hat{\mathbf{p}}$ such that the residual between the model and the data is small, is

$$
\begin{aligned}
S(\hat{\mathbf{p}}) & \approx S\left(\mathbf{p}^{*}\right)+\left[2 \mathbf{J}\left(\mathbf{p}^{*}\right)^{T} \mathbf{R}\left(\mathbf{p}^{*}\right)\right]\left(\hat{\mathbf{p}}-\mathbf{p}^{*}\right) \\
& +\frac{1}{2}\left(\hat{\mathbf{p}}-\mathbf{p}^{*}\right)^{T}\left[2 \mathbf{J}\left(\mathbf{p}^{*}\right)^{T} \mathbf{J}\left(\mathbf{p}^{*}\right)\right]\left(\hat{\mathbf{p}}-\mathbf{p}^{*}\right),
\end{aligned}
$$

where $\mathbf{J}$ is the $R \times p$ Jacobian of $\mathbf{R}$ and the terms in brackets are the Jacobian and approximate Hessian of the error function, respectively. One method for finding the minimum of a function is to search for a solution such that its gradient is zero and its Hessian is positive definite. Thus the minimization problem is converted to solving the following system of equations,

$$
\mathbf{0}=\mathbf{J}(\hat{\mathbf{p}})^{T} \mathbf{R}(\hat{\mathbf{p}})
$$

subject to the constraint that its approximate Jacobian $\mathbf{J}(\hat{\mathbf{p}})^{T} \mathbf{J}(\hat{\mathbf{p}})$ must be positive definite. The requirement that the Jacobian must be positive definite at a solution not only results from the need to find a minimum to the error function, but it also is required to find unique parameters. If the Jacobian is rank deficient, or close to singular, it is possible to define sets of parameters that will yield nearly identical fits. To solve Eqn. (39), MATLAB's fsolve algorithm was used. A starting guess for the parameters is also needed, so except for a single numerical experiment, the nominal parameters were selected as the initial guess. The convergence capabilities of this method are not of primary interest, so the use of the nominal parameters in general decreases the number of iterations needed for convergence. In the one experiment where the nominal parameters were not used, a different set was specifically used to address convergence issues. While how robust a method is to poor initial parameter estimates is important, the selection of the nominal parameters as the initial guess allows ones to indirectly investigate the biases of the estimated parameters.

Note that in the numerical experiments, the case that $\epsilon$ is set to 1 and the rest of the parameters were scaled appropriately was considered. It was found, that for this case, this Jacobian is nearly singular such that standard 
nonlinear equation solvers could not be used. An application of $\mathrm{Gu}$ and Eisenstat's strong rank revealing QR algorithm [54], as implemented in [55], showed that the parameter mostly responsible for this singularity is $Q$. In the numerical experiments, $Q$ was selected to qualitatively match ones found from experiments, thus it might be possible to set $\epsilon$ to 1 for systems with lower $Q \mathrm{~s}$, or identically, systems with stronger damping. The application of this research, however, is for modeling micro/nanosystems with small damping, thus, the appropriate selection of $\epsilon$ is important.

While the numerical experiments in this section were conducted without simulating noise being added to the data, given the distribution of the noise added to the data, it is possible to estimate the mean and standard deviation of the estimates. Specifically, assuming that noise is added to the data from the same distribution, and that this distribution has a zero mean and standard deviation of $\sigma_{m}$, then under a few other assumptions, the details of which can be found in [53], it can be stated that the parameters found from the least-squares method are unbiased estimates of the actual parameters and that the covariance of the parameter estimates is

$$
\operatorname{Cov}(\hat{p})=\sigma_{m}^{2}\left[\mathbf{J}(\hat{\mathbf{p}})^{T} \mathbf{J}(\hat{\mathbf{p}})\right]^{-1}
$$

In practice, $\sigma_{m}$ is not known a priori. Thus, the unbiased estimate of $\sigma_{m}$, or $\hat{\sigma}_{m}$, is used, where

$$
\hat{\sigma}_{m}^{2}=\frac{S(\hat{\mathbf{p}})}{R-p}
$$

so in conjunction with the assumption that $R$ is large, confidence intervals for $\hat{p}$ can be calculated from a t-distribution with $R-p$ degrees of freedom. The authors of this work would like to note two issues with generating confidence intervals for the parameter estimates. The first is that while Monto Carlo experiments with small levels of noise have been conducted to validate that under the given assumptions, $\hat{p}$ and $\hat{\sigma}_{m}$ belong to the expected distributions, there can be limitations with using an approximate linearization method for calculating confidence intervals [56]. The second is that $\hat{\sigma}_{m}$ is an unbiased estimator assuming that the model is accurate. In the numerical experiments presented in this work, a case where $\hat{\sigma}_{m}$ is significantly greater than $\sigma_{m}$ can be indicative of an issue or issues with the associated sub-problems related to parametric system identification.

Much of the presented fitting method requires the Jacobian $\mathbf{J}$. This Jacobian is often found approximately, such as with finite difference methods, 
but it is possible to calculate the Jacobian directly using the implicit function theorem. While not shown in the previous section, it is possible to use the averaged equations to find a single polynomial equation for the steady-state amplitude by using the trigonometric identity

$$
1=\cos ^{2} \phi+\sin ^{2} \phi
$$

From the steady-state amplitude equation, which is of the form

$$
0=h(a, \hat{\mathbf{p}}),
$$

it is then possible to find the derivative of the steady-state amplitude with respect to a given parameter $p_{i}$ by

$$
\frac{d a}{d p_{i}}=-\frac{\partial h}{\partial p_{i}} / \frac{\partial h}{\partial a} .
$$

Figures 7, 8, and 10, which correspond to Cases 1, 2, and 3, respectively, show the harmonic balance solutions to Eqn. (19) with the nominal parameters and accounting for fifth-order nonlinearities, the first-order averaged solution with its fitted parameters, and the second-order averaged solution with its fitted parameters, in red, black and blue, respectively. In order to show how well the fits match the numerically generated data, green dots and magenta circles corresponding to the sampled harmonic balance solution for discrete forward and reverse sweeps of the excitation frequency, respectively, are included in the figures. The parameter estimates, and corresponding standard deviations, are given in Tables 2-4. In addition, these tables give the value of the error function for the estimated parameters and an estimate of the standard deviation of the noise. Note that no noise was added to the data in the numerical experiments in this section, thus the estimated noise is an artifact of issues associated with sub-optimal approaches to dealing with the sub-problems associated with parametric system identification.

For Case 1, as one might expect, the two fits based on the averaged solutions quantitatively match the data generated by the harmonic balance method. This is validated numerically, since the values of the estimated noise, which are given in Table 2, are much smaller than all of the numerically generated data (i.e. the relative error is very small). The limitations of the two fits, however, are the significant biases between the estimated and nominal parameters. Thus, while the fitted parameters could be used 
Table 2: A table of the nominal system parameters, parameter estimates, and error functions resulting from fitting the generated data to the first- and second-order averaged solutions for Case 1.

\begin{tabular}{cccc} 
& Nominal & $O(\epsilon)$ Avg. & $O\left(\epsilon^{2}\right)$ Avg. \\
$Q$ & $2.12245 \times 10^{-1}$ & $2.11719 \times 10^{-1}$ & $2.12522 \times 10^{-1}$ \\
& & $\pm 1.44262 \times 10^{-4}$ & $\pm 1.09806 \times 10^{-7}$ \\
$k_{3}$ & 3.27127 & $\mathrm{NA}$ & 5.22693 \\
& & & $\pm 2.54069 \times 10^{-4}$ \\
$\beta$ & 4.59677 & $\mathrm{NA}$ & 7.53027 \\
& & & $\pm 3.81350 \times 10^{-4}$ \\
$\eta_{1}$ & $6.12500 \times 10^{4} \mathrm{~A}^{-1}$ & $6.12476 \times 10^{4} \mathrm{~A}^{-1}$ & $6.12500 \times 10^{4} \mathrm{~A}^{-1}$ \\
& & \pm 9.03395 & $\pm 5.97615 \times 10^{-3} \mathrm{~A}^{-1}$ \\
$\gamma_{3}$ & $7.54910 \times 10^{-4}$ & $-1.05212 \times 10^{-3}$ & $1.66898 \times 10^{-3}$ \\
& & $\pm 4.39020 \times 10^{-4}$ & $\pm 3.44050 \times 10^{-7}$ \\
$\alpha_{3}$ & $6.20270 \times 10^{-1}$ & $6.24179 \times 10^{-1}$ & $6.20269 \times 10^{-1}$ \\
& & $\pm 1.92456 \times 10^{-4}$ & $\pm 5.11481 \times 10^{-7}$ \\
$S(\hat{\mathbf{p}})$ & $\mathrm{NA}$ & $7.36553 \times 10^{-5}$ & $3.19327 \times 10^{-11}$ \\
$\hat{\sigma}_{m}$ & $\mathrm{NA}$ & $7.05458 \times 10^{-4}$ & $4.66078 \times 10^{-7}$ \\
\hline
\end{tabular}

in models that quantitatively match experimental results, these parameters lack physical significance. However, given that in experiments one might conduct trials with different excitation amplitudes, or more practically, a model that is applicable for multiple excitation amplitudes is of greater utility, an excitation-dependent bias significantly complicates the selection of parameters needed to even qualitatively match the experimental results. The largest relative difference between the estimated and nominal parameters for both fits comes from the $\gamma_{3}$ estimate, which is related to nonlinear damping effects. The estimate for $\gamma_{3}$ from the first-order averaged solution is particu- 


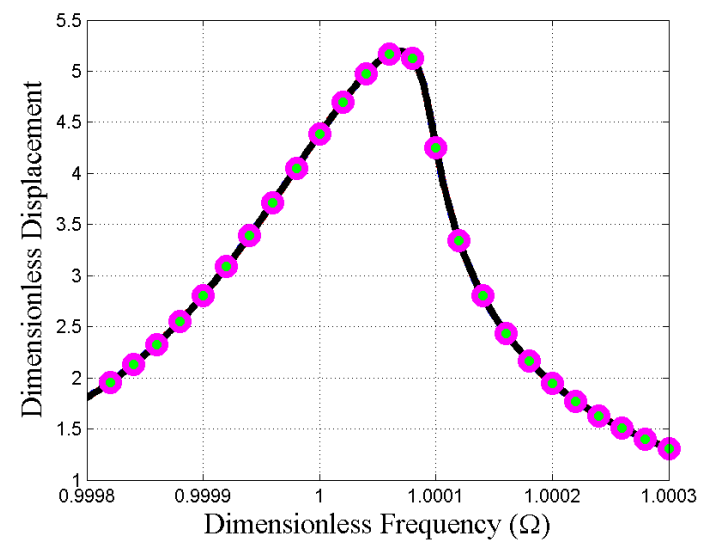

Figure 7: The harmonic balance solution to Eqn. (19) with the nominal parameters and accounting for fifth-order nonlinearities, the first-order averaged solution with its fitted parameters, and the second-order averaged solution with its fitted parameters are shown in red, black and blue, respectively, for Case 1. Note that the green dots and magenta circles correspond to the discretely sampled harmonic balance solution for forward and reverse sweeps of the excitation frequency, respectively.

larly troublesome, as it is negative. ${ }^{1}$ The significance of nonlinear damping for excitations in that range, however, is small, which is one explanation as to why these fitting methods yield poor estimates.

Comparing the results of the two fits, the model based on the second-order averaged solution performs better. In Case 1, this solution yielded estimates with smaller biases. Using the results from Case 2, which are shown in Table 3 , in conjunction with the results from Case 1, one might extrapolate that between trials, the biases of the second-order averaged model parameters vary less than the biases of the first-order averaged model parameters. That is, provided the error function is small, with the second-order averaged solution as the model, one might only need to conduct a few trials to generate a parametric model that quantitatively matches experimental results. This is important when testing with micro/nanosystems when a lock-in detection

\footnotetext{
${ }^{1} \mathrm{~A}$ negative third-order nonlinear damping coefficient corresponds to a system with self-excitation capabilities [57], or a condition where damping mechanisms can add energy to the system. Thus, the issue of estimating a negative third-order nonlinear damping coefficient is twofold, in that besides the parameter estimate being significantly biased, the estimate incorrectly predicts a physical phenomena not found in the nominal system.
} 
method is used, since it can take a significant amount of time to conduct a single trial. This might not be possible when the first-order averaged solution is employeed as the approximate solution, since its corresponding error function for Case 2 was large.

For the Case 2 responses, which are shown in Fig. 8, the differences between averaged solutions and the harmonic balance solution may appear to be small, but the second-order averaged solution fits the data much more closely. To show the differences between the various responses, the same responses shown in Fig. 8 are shown in Fig. 9, but over a smaller range of excitation frequencies. It is important to note that for Case 2, the parameters for the first-order averaged solution do not actually minimize the error function, since the error of the approximate Hessian in this case is significant. It might be possible for the first-order averaged solution to more closely match the harmonic balance solution if the exact Hessian was used, but fundamentally the first-order averaged solution poorly models the numerical experiment.

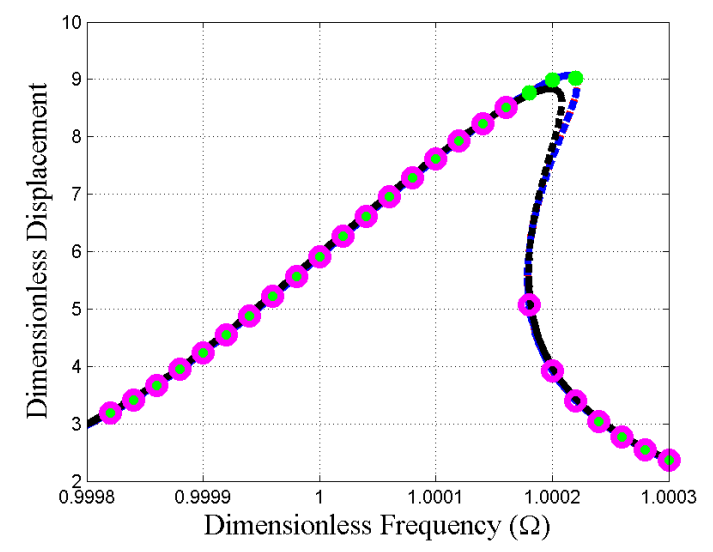

Figure 8: A figure similar to Fig. 7, except for Case 2. Note that for Case 2, since the amplitude of the current supplied to the system is greater than the critical current, stable and unstable solutions are present in the steady-state response, and are denoted by solid and dashed lines, respectively.

As expected, the error of second-order averaged solution increases as the excitation increases. Case 3 is an example of this. Unlike the two previous cases, the averaged solutions for Case 3, shown in Fig. 10, do not as closely mirror the harmonic balance solution. This is highlighted in Fig. 11. In particular, the averaged solutions have the largest differences near their respective maximum amplitudes. This error can be described numerically, as 
Table 3: A table similar to Table 2, but for Case 2.

\begin{tabular}{cccc} 
& & & \\
$Q$ & Nominal & $O(\epsilon)$ Avg. & $O\left(\epsilon^{2}\right)$ Avg. \\
& $2.12245 \times 10^{-1}$ & $2.22576 \times 10^{-1}$ & $2.13102 \times 10^{-1}$ \\
& & $\pm 7.35502 \times 10^{-2}$ & $\pm 6.89473 \times 10^{-7}$ \\
$k_{3}$ & 3.27127 & $\mathrm{NA}$ & 5.22831 \\
& & $\mathrm{NA}$ & $\pm 1.73339 \times 10^{-4}$ \\
$\beta$ & 4.59677 & & 7.53234 \\
& & & $\pm 2.60450 \times 10^{-4}$ \\
$\eta_{1}$ & $6.12500 \times 10^{4} \mathrm{~A}^{-1}$ & $6.14063 \times 10^{4} \mathrm{~A}^{-1}$ & $6.12499 \times 10^{4} \mathrm{~A}^{-1}$ \\
& & $\pm 3.66949 \times 10^{-2} \mathrm{~A}^{3}$ & $\pm 3.28912 \times 10^{-2} \mathrm{~A}^{-1}$ \\
$\gamma_{3}$ & $7.54910 \times 10^{-4}$ & $1.90501 \times 10^{-2}$ & $1.68953 \times 10^{-3}$ \\
& & $\pm 7.66647 \times 10^{-2}$ & $\pm 6.94497 \times 10^{-7}$ \\
$\alpha_{3}$ & $6.20270 \times 10^{-1}$ & $6.22351 \times 10^{-1}$ & $6.20264 \times 10^{-1}$ \\
& & $\pm 4.03425 \times 10^{-2}$ & $\pm 9.41794 \times 10^{-7}$ \\
$S(\hat{\mathbf{p}})$ & $\mathrm{NA}$ & $3.12047 \times 10^{1}$ & $2.26869 \times 10^{-9}$ \\
$\hat{\sigma}_{m}$ & $\mathrm{NA}$ & $4.59176 \times 10^{-1}$ & $3.92852 \times 10^{-6}$ \\
\hline
\end{tabular}

the estimated noises, which are given in Table 4, are on par with the numerically generated data. The biases of the parameters in Table 4 may appear to be surprisingly small, but this is due to the use of the nominal parameters as the starting guesses. This particular case was also conducted using a finite-difference method for the Jacobian, since the previously mentioned approximate Hessian is inaccurate due to a significant residual. The finitedifference version of this experiment was conducted with an $N=5$ harmonic balance solution as the benchmark, since for $N=10$ it failed to converge after $10^{5}$ iterations. The Jacobians corresponding to both the first- and secondorder averaged solutions were rank-deficient, which at best suggests sets of parameters for both models can be defined that can satisfy Eqn. (39) and at worst suggests no solutions exist. One might be able to argue that the averaged solutions based on the third-order model qualitatively match the 


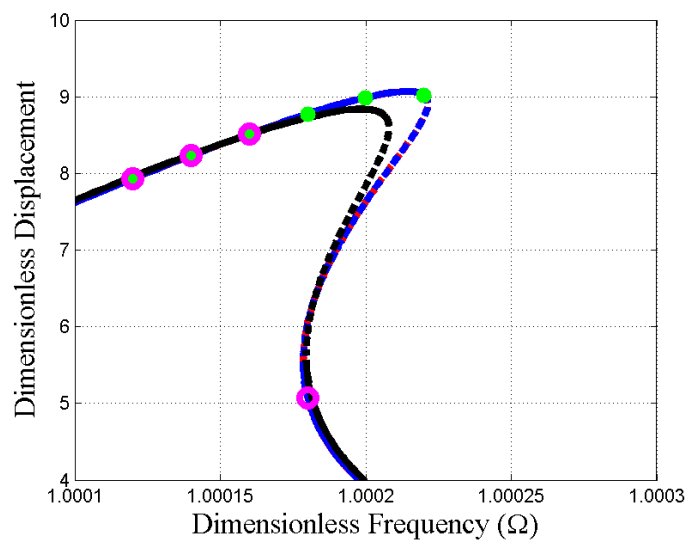

Figure 9: The same responses shown in Fig. 8, but over a smaller range of excitation frequencies so as to highlight the differences between the various responses.

harmonic balance solution. The danger of such a statement is that this type of incorrect correlation between qualitative and quantitative match leads to parameter estimates that do not correspond to the system's reduced-order model, and thus lack any physical significance. In addition, the authors are unaware of statistical methods that can be applied in a case when the noisefree residual is large, so these parameters also lack statistical significance. Thus, besides the difficulties associated with finding parameter estimates, in general, a model that produces significant residuals between it and a more accurate one and/or has a corresponding error function with a rank-deficient Hessian will yield parameter estimates that lack uniqueness and significance, in both a physical and statistical sense.

\section{Reduced-Order Model Selection: Fitting to Model-Generated Data with Harmonic Balance Solutions}

In the previous section, two system identification methods based on averaging were presented. While these methods are quite computationally efficient, and generating steady-state responses to slow forward and reverse sweeps in excitation frequency is simple, they can only be applied successfully for a limited range of excitations. In addition, some of the parameter estimates are strongly biased. As mentioned in the introduction and Section 5, there are many cases where the first-order averaged solution effectively couples parameters, which in turn complicates the design, characterization, 
Table 4: A table similar to Table 2, but for Case 3 .

\begin{tabular}{cccc}
\hline & Nominal & $O(\epsilon)$ Avg. & $O\left(\epsilon^{2}\right)$ Avg. \\
$Q$ & $2.12245 \times 10^{-1}$ & $2.16252 \times 10^{-1}$ & $2.05738 \times 10^{-1}$ \\
& & $\pm 2.15475 \times 10^{-1}$ & $\pm 1.34565 \times 10^{-1}$ \\
$k_{3}$ & 3.27127 & $\mathrm{NA}$ & 3.27231 \\
& & $\mathrm{NA}$ & $\pm 2.08386 \times 10^{1}$ \\
$\beta$ & 4.59677 & & 4.59382 \\
& & & $\pm 3.13021 \times 10^{1}$ \\
$\eta_{1}$ & $6.12500 \times 10^{4} \mathrm{~A}^{-1}$ & $6.12528 \times 10^{4} \mathrm{~A}^{-1}$ & $6.15264 \times 10^{4} \mathrm{~A}^{-1}$ \\
& & $\pm 9.90679 \times 10^{3} \mathrm{~A}^{3}$ & $\pm 6.09218 \times 10^{3} \mathrm{~A}^{-1}$ \\
$\gamma_{3}$ & $7.54910 \times 10^{-4}$ & $1.09870 \times 10^{-2}$ & $-1.29205 \times 10^{-4}$ \\
& & $\pm 1.16776 \times 10^{-1}$ & $\pm 7.14132 \times 10^{-2}$ \\
$\alpha_{3}$ & $6.20270 \times 10^{-1}$ & $6.27085 \times 10^{-1}$ & $6.29295 \times 10^{-1}$ \\
& & $\pm 7.13136 \times 10^{-2}$ & $\pm 9.75496 \times 10^{-2}$ \\
$S(\hat{\mathbf{p}})$ & $\mathrm{NA}$ & $3.17104 \times 10^{2}$ & $1.11675 \times 10^{2}$ \\
$\hat{\sigma}_{m}$ & $\mathrm{NA}$ & 1.46376 & $8.71603 \times 10^{-1}$ \\
\hline
\end{tabular}

and implementation of such systems. Thus, there is need for characterization methods that can uniquely identify parameters with small biases. This section explores the use of harmonic-balance-based methods that seek to accomplish both tasks.

Section 3 describes the method used to generate the data for the numerical experiments. In order to produce accurate approximate solutions to Eqn. (19), a high number of harmonics were used. Given the difficulty of explicitly generating the functions that must be solved in order to find the $C_{n}$ and $S_{n}$, a discrete Fourier transform method was used. Based on the results of the residual convergence test shown in Fig. 3, it is possible that a lower-order approximation could be used to produce steady-state responses with relatively smaller residuals between the lower-order approximation and the higher-order approximation than between the averaged solutions and the 


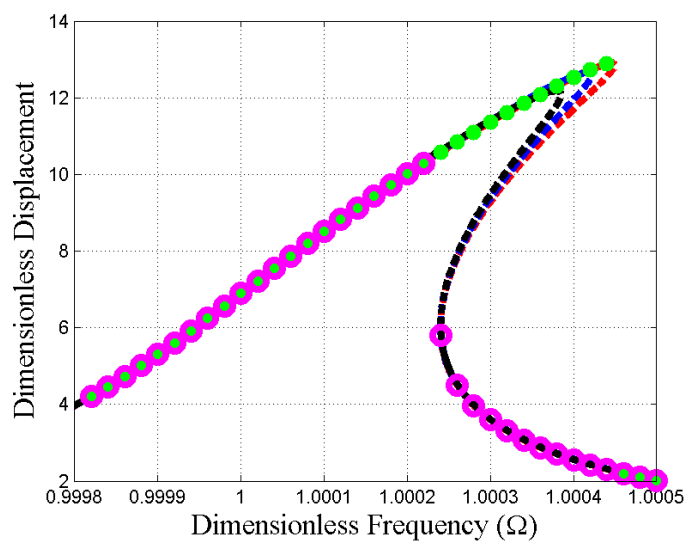

Figure 10: The Case 3 steady-state responses.

higher-order approximation. Thus, in this section, two $N=3$ harmonic balance solutions, one for the third-order reduced order model and one for the fifth-order reduced order model, which are based on solving a system of explicitly-defined equations, are used to facilitate parametric system identification. An important reason that one would want to use explicitly-defined equations is that it allows one to use the implicit function theorem to calculate solution sensitivities.

In order to generate the system of equations used for the harmonic balance solution, MATHEMATICA was used to perform the various substitutions, factorizations, and simplifications needed. As referenced in Section 6, the Jacobian of the steady-state solution is needed, so the higher-order version of the implicit function theorem was invoked. Given a system of equations of the form

$$
\mathbf{0}=\mathbf{H}(\hat{\mathbf{C S}}, \hat{\mathbf{p}}),
$$

where the components of the vector function $\mathbf{H}$ are the equations that need to be solved and $\hat{\mathbf{C S}}$ denotes a vector of the $C_{n}$ and $S_{n}$ concatenated together, the sensitivities of the $C_{n}$ and $S_{n}$ to a particular component of $\hat{\mathbf{p}}$ can be found by solving the following linear system of equations

$$
\left[\frac{\partial \mathbf{H}}{\partial \hat{\mathbf{C S}}}\right] \frac{d \hat{\mathbf{C S}}}{d p_{i}}=-\frac{\partial \mathbf{H}}{\partial p_{i}} .
$$

Note that the term on the left and in brackets is simply the Jacobian of the vector function with respect to the $C_{n}$ and $S_{n}$. 


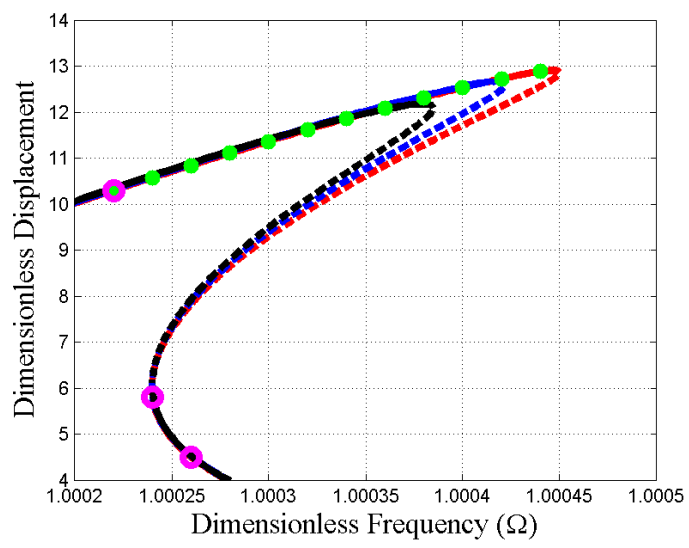

Figure 11: The same responses shown in Fig. 10, except over a smaller range of excitation frequencies.

When numerical experiments were conducted with the method that employs the fifth-order reduced-order model on the cases presented in the previous section, this method failed due to a rank deficiency of the error function's approximate Hessian. This failure was not caused by the relative error of the lower-order harmonic balance solution, but by deficiencies of the isolated cases' sensitivity to the parameters. Using the same strong rank revealing algorithm as referenced earlier, the rank deficiencies for the Cases 1 and 2 were caused by $\beta, \nu_{1}$, and $\nu_{2}$, and for Case 3 it was caused by just $\beta$ and $\nu_{1}$. In order to produce a full rank approximate Hessian, all three cases were considered simultaneously. The estimated parameters are shown in Table 5 and the fitted responses are shown in Fig. 12. In Fig. 12, the $N=10$ responses used to generate the numerical experiment's data are shown in red, the fitted responses from the $N=3$ harmonic balance solution corresponding the third-order reduced-order model are shown in black, and the fitted responses from the $N=3$ harmonic balance solution corresponding the fifth-order reduced-order model are shown in blue. As in the previous section, the numerical experiment's data corresponding to responses from forward and reverse sweeps in frequency are shown with green dots and magenta circles, respectively. However, in order to not only demonstrate these methods in the presence of disturbances, but also a model selection method, a small amount of noise was added to the data. That is, random values from a normal distribution with zero mean and standard deviation of $5 \times 10^{-3}$ were 
added to the numerically generated data. The estimated parameters for both methods, as well as their corresponding error functions and noise estimates, are shown in Table 5. Finally, in regards to how the numerical experiments were setup, while the starting parameter estimates for the $N=3$ harmonic balance solution corresponding the fifth-order reduced order model were the nominal parameter estimates, some of the starting parameter estimates for the $N=3$ harmonic balance solution corresponding the third-order reduced order model were slightly perturbed to address issues associated with bias and convergence. That is, the initial estimates for $k_{3}$ and $\beta$ were set as 5.22819 and 7.53215 , respectively, as these methods are very sensitive to poor initial parameter estimates.

In order to put the aforementioned numerical experiments in context, a brief review of parameter estimation in the absence of noise can be illustrative. When the $N=3$ harmonic balance solution corresponding to the fifth-order reduced-order model was used, to within the precision reported in the tables in this work, the estimated parameters were equal to the nominal parameters. This was due to the bias of this estimation method being relatively small. Furthermore, the noise estimate, $\hat{\sigma}_{m}$, was $8.03322 \times 10^{-12}$ was very small. For the parameters estimated with the third-order reduced-order model, the bias of the estimates was akin to those in Table 3 for the secondorder averaged solution and the noise estimate was $1.64532 \times 10^{-3}$. Thus, when the standard deviation of the noise present in a given experiment is less than the previously given value, one would expect that the fifth-order reduced-order model provides a better fit. Conversely, when the level of noise is much greater than this value, it may not be possible for one to determine which model provides a better fit.

With the interjection of noise, the estimated parameters corresponding to both models provide an exemplar case of the classic bias-variance issue [24]. While the confidence intervals for the parameters corresponding to the fifth-order, reduced-order model encompass the nominal values, most of these intervals span several orders of magnitude. The relative uncertainties of the parameters for the third-order reduced-order model were much smaller, but a bias similar to the noise-free experiment was still present. The fundamental dilemma this experiment revealed, however, was that neither model provides an optimal means of identify the underlying physical phenomena.

Provided one has parameter estimates for both models in hand, a logical question to ask is which model is better. Ignoring problems associated with the physical significance of the parameter estimates, as both models poorly 
address this issue, one could conduct hypothesis tests to ascertain if the parameters unique to the fifth-order reduced order model are zero. Alternatively phrased, one seeks to determine if the higher-order model fits the data better, in a statistical sense, than the lower-order model . In general, a highorder model will produce a smaller error function than a lower-order model, but this test seeks to answer the question of if this difference is statically significant. One test statistic one could use is the following,

$$
\hat{F}=\frac{S\left(\hat{\mathbf{p}}_{1}\right)-S(\hat{\mathbf{p}})}{S(\hat{\mathbf{p}})} \frac{R-p}{p-p_{1}},
$$

where, as before, $\hat{\mathbf{p}}$ is a vector of the parameter estimates corresponding to the higher-order model and $\hat{\mathbf{p}}_{1}$ is a vector of the parameter estimates corresponding to the lower-order model [53]. Note that in order to calculate this test statistic, one needs to estimate parameters for both models. Assuming that the null hypothesis is true, or that the parameters that are unique to the high-order model are zero, $\hat{F}$ is approximately $F$-distributed with $p-p_{1}$ and $R-p$ degrees of freedom, where $p_{1}$ is the length of $\hat{\mathbf{p}}_{1}$. For the numerical experiments reported in Table $5, \hat{F}=13.9611$. The associated probability of this occurrence (i.e. $P(F>\hat{F})$ ) is $9.66659 \times 10^{-11}$, which is sufficiently low that even most conservative significance levels would reject the null hypothesis. This might lead one to conclude that the fifth-order model is the better model. This assessment, however, is based on how the experiment was setup. With a well designed experiment, a dimensionless noise level on the order of $5 \times 10^{-3}$ or smaller is obtainable. However, if experimental issues arise such that the dimensionless noise level is larger, the additional fidelity of the fifth-order reduced-order model may be unwarranted. When the level of noise was increased to $5 \times 10^{-2}, \hat{F}=6.77104 \times 10^{-1}$ and the associated probability of this occurrence was $6.08117 \times 10^{-1}$. Thus, for the case of a poorly-designed experiment, one would accept the null hypothesis.

The larger point that this section seeks to address is that the determination of the accuracy a given parametric system identification is relative. The previous section sought to demonstrate that fundamentally, certain approximately solutions are flawed. However, once that issue has been addressed, the determination of the appropriate reduced-order model is contingent on how well it compares to other reduced-order models. In the numerical experiments conducted in this section, the only parametric system identification issue considered was noise, and it was shown that changing the level of the noise can change which model is accepted. With poor experimental design, or in the 
case that the micro/nanosystem is operating in a noisy environment, a lowfidelity model may be sufficient. Conversely, with a well designed experiment, a high-fidelity model is needed to mitigate issues associated with parameter estimate bias. Other challenges one might encounter in an experimental context are issues associated with the data being biased due to how the lock-in amplifier was setup or how close the amplitude of the microresonator is to steady-state. Comparing both lower- and higher-order models against experimentally acquired results could also help one to identify these experimental issues. Thus, there is a strong utility in comparing reduced-order models, as besides providing a basis for assessment, doing so can also identify issues with the other associated sub-problems. Hopefully, this dilemma and the ones mentioned in previous sections will inspire further research on the parametric system identification of resonant micro/nanosystems that operate in a nonlinear response regime.

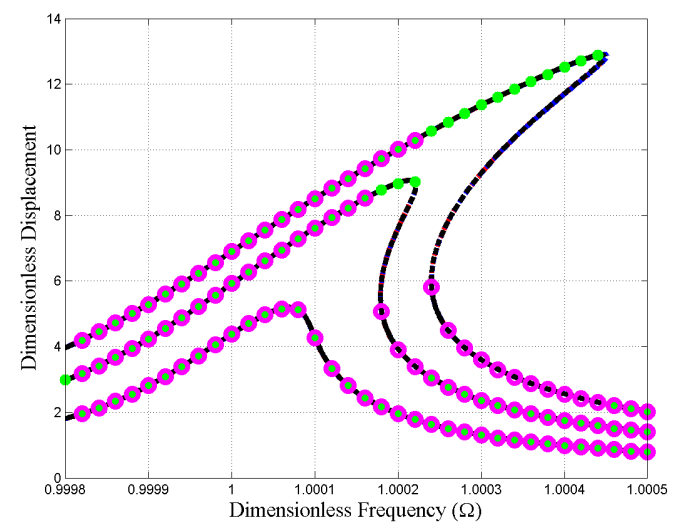

Figure 12: Overlays of the nominal responses with the responses generated from the estimated parameters. The nominal responses correspond to the $N=10$ harmonic balance solution described in Section 3 are shown in red. The fitted responses corresponding to the $N=3$ harmonic balance solution with the third-order and fifth-order reduced order model are shown in black and blue, respectively. As in the previous section, the numerical experiment's data corresponding to responses from forward and reverse sweeps in frequency are shown with green dots and magenta circles, respectively.

\section{Conclusions and Future Directions}

Due to experimental constraints, parametric system identification of micro/nanosystems can be challenging. Specifically, since a lock-in amplifier is 
used, only the amplitude and phase of the measured response at a single frequency can be measured. Thus, many of the methods used to characterize macroscale systems are not applicable. Methods that can be used, however, are methods based on fitting the experimentally-recovered response to an approximate solution. With this is mind, it is proposed that parametric system identification of resonant micro/nanosystems operating in a nonlinear regime be decomposed into four sub-problems: nonparametric system identification, reduced-order modeling, approximate response calculation, and nonlinear least-squares parameter estimation. Due to the issues related to each of these sub-problems, which were discussed in this work, this effort focused on the theoretical aspects of the last three sub-problems. The primary results recovered are:

- At excitations close to the critical one, or at ones which hysteretic behavior can be observed in the steady-state response, discrepancies between third- and fifth-order models can prohibit accurate parameter estimation. This result is not specifically constrained to the previouslyreferenced model orders, and is more generally regarding model fidelity, but third-order models are often used in attempts to explain observed responses.

- Approximate solutions that one could derive using common perturbation methods can yield significant residuals between the approximate solution and a more accurate one, which again can prohibit parameter estimation. If used judiciously, however, these perturbation-based methods can be used to produce quantitatively-accurate fits. The caveat with these fits is that the parameter estimates may be significantly biased.

- Approximate solutions, or those that could be found from either perturbation methods like the method of averaging or the harmonic balance method, can effectively couple parameters together, which besides introducing problems with standard least-squares methods, inhibits unique parameter estimation. In this work, an example of effectively coupling geometric and inertial nonlinearities was presented. Other notable ones can arise due to the interplay between actuation and mechanical nonlinearities or cases when different phenomena are exploited for actuation and sensing. 
- Two harmonic-balance-based parameter estimation methods were presented. While both of these methods addressed some of the limited dynamic range issues that perturbation-based methods can have, the interjection of noise provided a classic example of the bias-variance issue. That is, while the higher-order model was able to estimate the parameters with small biases, the variability of these estimates was very large. Likewise, while the variability of the parameter estimates from the lower-order model was relatively much smaller, these parameter estimates were significantly biased. Thus, neither model provides an optimal means of identifying the underlying physical phenomena. However, hypothesis testing may provide a means of assessing which model provides a better fit to the data, as fundamentally, the selection of one model over another is based on relative performance. These tests may also reveal issues with the other associated sub-problems.

Thus, while a framework for the characterization of micro/nanosystems operating in a nonlinear response regime is presented, there is still significant work to be done.

\section{Acknowledgments}

This material is based in part upon work supported by the National Science Foundation under Grant Number 0846385. Any opinions, findings, and conclusions or recommendations expressed in this material are those of the authors and do not necessarily reflect the views of the National Science Foundation. In addition, this work would not have been possible with out the efforts of A. G. Hunkler.

\section{Appendix A. Justification for Nonlinear Damping}

The need for the model to account for nonlinear damping is based on experimental results, which are shown in Fig. A.13, and theoretical results, which are subsequently discussed. In these experiments, the excitation frequency was either increased (forward sweep) or decreased (reverse sweep) in small steps near the first natural frequency of the cantilever, in order to show the hysteretic response of the system. Note that data from forward and reverse sweeps are denoted by dots and circles, respectively. This figure shows that the maximum normalized steady-state velocity amplitude, where 
the normalized response is defined as the steady-state velocity amplitude divided by the amplitude of the excitation voltage, decreases as the excitation voltage increases.

Contrary to claims in previously-published works [21], the authors would like to point out that geometric and inertial nonlinearities can cause the maximum normalized response to decrease as the forcing amplitude increases. Using the model, which accounts for geometric, inertial, and damping nonlinearities up to fifth-order, and the harmonic balance method from Sections 2 and 3, respectively, and the nominal parameters of the system, Fig. A.14 shows the maximum normalized steady-state amplitude verses the normalized current. The normalized current is defined as the product of the amplitude of the supplied current and the forcing term $\epsilon \eta_{1}$, and the maximum normalized steady-state amplitude is the maximum steady-state amplitude divided by the normalized current. Note that this forcing term $\epsilon \eta_{1}$ is dependent on the geometry of the microcantilever and the local magnetic field, and arises from the presented reduced-order model derivation. The red and blue curves in Fig. A.14 are for the cases where nonlinear damping is not and is present, respectively. Clearly the effect of the input current on the maximum normalized amplitude of the nonlinearly damped system is more significant, but the maximum normalized amplitude of the linearly damped system is not independent of the input current, as is incorrectly stated in [21].

More importantly, for the nonlinearly-damped system, above a threshold normalized current, the maximum normalized amplitude tends to linearly decrease, which is consistent with the experimental results in Fig. A.13. This threshold is directly dependent upon the strength of the nonlinear damping present in the system, so general conditions for when to consider nonlinear damping cannot be provided. However, assuming that the presented model is accurate, Fig. A.14 suggests that nonlinear damping must be considered for excitations close to, or greater than, the critical one. Thus, the authors of this work judiciously, but cautiously, argue that based on the experimental and theoretical results in Fig. A.13 and A.14, respectively, nonlinear damping is present and must be accounted for in the microcantilever model.

In regards to the actual form of the nonlinear damping model, while the selection of a Kelvin-Voigt material model may seem arbitrary, recent works have used this model to account for nonlinear damping [34, 36]. Fung [35] also argues that irreversible heat generation suggests a generalized Maxwell material model, thus failure to account for this effect would violate the second law of thermodynamics. The generalized Maxwell material model accounts 
for effects related to creep, but if creep is ignored since large static forces are not applied to the cantilever, the generalized Maxwell material model reduces to the Kelvin-Voigt material model. Moreover, a strong motivating reason for the selection of the Kelvin-Voigt material model is that it allows one to build upon efforts that have assumed a linear elastic material model. That is, by separating the total moment applied to the beam into conservative and nonconservative moments, published models that just consider the conservative moment only need to be augmented to account for the nonconservative moment.

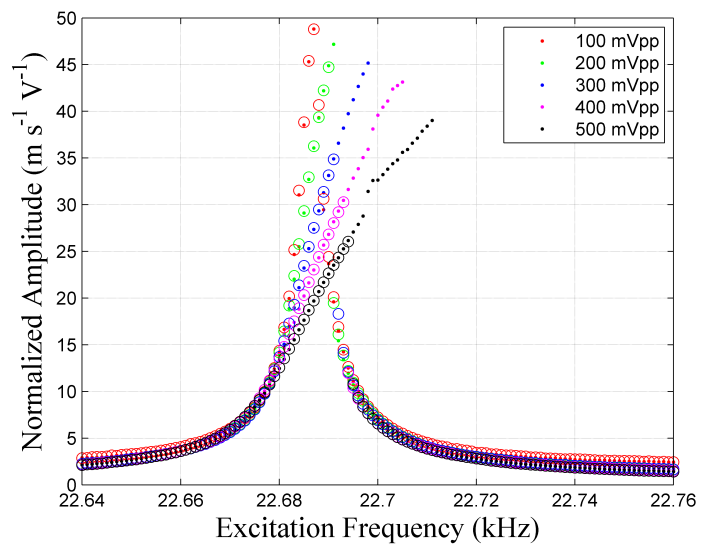

Figure A.13: The amplitude of the steady-state velocity of the device shown in Fig. 1, normalized with respect to the amplitude of the excitation voltage.

\section{Appendix B. Integral Expressions for Nondimensional Parame- ters in Eqn. (19)}

In the following appendix, the integral expressions for the parameters in Eqn. (19) are given in Tables B.6 and B.7

\section{References}

[1] Senturia SD. Microsystem Design. 1st ed.; Norwell, MA: Kluwer Academic Publishers; 2000.

[2] Liu C. Foundations of MEMS. Upper Saddle River, NJ: Pearson/Prentice Hall; 2006. 


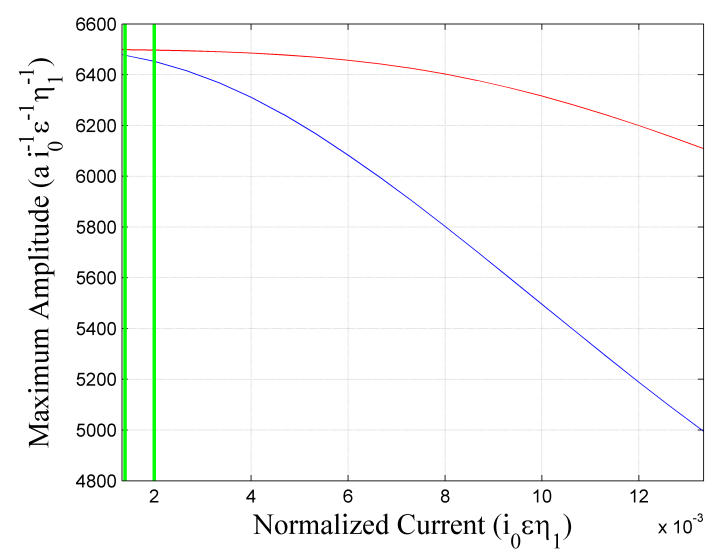

Figure A.14: The maximum normalized steady-state amplitude responses for the presented model with the nominal parameters, calculated using the presented harmonic balance method. Note that red and blue are used to denote responses without and with nonlinear damping, respectively. For reference, the green vertical lines, from left to right, correspond to the normalized currents for Cases 2 and 3.

[3] Waggoner PS, Craighead HG. Micro- and nanomechanical sensors for environmental, chemical, and biological detection. Lab Chip 2007;7(10):1238-55.

[4] Greywall DS. Micromechanical RF filters excited by the Lorentz force. Journal of Micromechanics and Microengineering 1999;9(1):78-84.

[5] Greywall DS, Yurke B, Busch PA, Pargellis AN, Willett RL. Evading amplifier noise in nonlinear oscillators. Physical Review Letters 1994;72(19):2992-5.

[6] Kozinsky I, Postma HWC, Bargatin I, Roukes ML. Tuning nonlinearity, dynamic range, and frequency of nanomechanical resonators. Applied Physics Letters 2006;88(25):253101.

[7] Kerschen G, Worden K, Vakakis AF, Golinval JC. Past, present and future of nonlinear system identificaiton in structural dynamics. Mechanical Systems and Signal Processing 2006;20(3):505-92.

[8] Sazonova V, Yaish Y, Ustunel H, Roundy D, Arias TA, McEuen PL. A tunable carbon nanotube electromechanical oscillator. Nature 2004;431(7006):284-7. 
[9] Gouttenoire V, Barois T, Perisanu S, Leclercq JL, Purcell ST, Vincent $\mathrm{P}$, et al. Digital and FM demodulation of a doubly clamped single-walled carbon-nanotube oscillator: Towards a nanotube cell phone. Small 2010;6(9):1060-5.

[10] Yu L, Pajouhi H, Nelis MR, Rhoads JF, Mohammadi S. Tunable, dualgate, silicon-on-insulator (SOI) nanoelectromechanical resonators. IEEE Transactions on Nanotechnology 2012;11(6):1093-9.

[11] Sabater AB, Kumar V, Mahmood A, Rhoads JF. On the nonlinear dynamics of electromagnetically-transduced microresonators. Journal of Microelectromechanical Systems 2013;22(5):1020-31.

[12] Hanagud SV, Meyyappa M, Craig JI. Method of multiple scales and identification of nonlinear structural dynamic systems. AIAA Journal $1985 ; 23(5): 802-7$.

[13] Nayfeh AH. Parametric identification of nonlinear dynamic systems. Computers \& Structures 1985;20(13):487-93.

[14] Krauss RW, Nayfeh AH. Experimental nonlinear identification of a single mode of a transversely excited beam. Nonlinear Dynamics 1999;18(1):69-87.

[15] Doughty TA, Davies P, Bajaj AK. A comparison of three techniques using steady state data to identify non-linear modal behavior of an externally excited cantilever beam. Journal of Sound and Vibration 2002;249(4):785-813.

[16] Malatkar P, Nayfeh AH. A parametric identification technique for singledegree-of-freedom weakly nonlinear systems with cubic nonlinearities. Journal of Vibration and Control 2003;9(3-4):317-36.

[17] Dick AJ, Balachandran B, DeVoe DL, Mote Jr. CD. Parametric identification of piezoelectric microscale resonators. Journal of Micromechanics and Microengineering 2006;16(8):1593-601.

[18] Liu J, Martin DT, Kadirvel K, Nishida T, Cattafesta L, Sheplak M, et al. Nonlinear model and system identification of a capacitive dual-backplate MEMS microphone. Journal of Sound and Vibration 2008;309(1-2):27692. 
[19] Villanueva LG, Karabalin RB, Matheny MH, Chi D, Sader JE, Roukes ML. Nonlinearity in nanomechanical cantilevers. Physical Review B 2013;87(2):024304.

[20] Ruzziconi L, Younis MI, Lenci S. Parameter identification of an electrically actuated imperfect microbeam. International Journal of NonLinear Mechanics 2013;57:208-19.

[21] Imboden M, Williams O, Mohanty P. Nonlinear dissipation in diamond nanoelectromechanical resonators. Applied Physics Letters 2013;102(10):103502.

[22] Imboden M, Williams OA, Mohanty P. Observation of nonlinear dissipation in piezoresistive diamond nanomechanical resonators by heterodyne down-mixing. Nano Letters 2013;13(9):4014-9.

[23] Singh R, Davies P, Bajaj AK. Identification of nonlinear and viscoelastic properties of flexible polyurethane foam. Nonlinear Dynamics 2003;34(34):319-46.

[24] Hastie T, Tibshirani R, Friedman J. The Elements of Statistical Learning: Data Mining, Inference, and Prediction. Springer; 2011.

[25] Paci D, Pieri F, Toscano P, Nannini A. A CMOS-compatible, magnetically actuated resonator for mass sensing applications. Sensors and Actuators B: Chemical 2008;129(1):10-7.

[26] Requa MV, Turner KL. Electromechanically driven and sensed parametric resonance in silicon microcantilevers. Applied Physics Letters 2006;88(26):263508.

[27] Requa MV, Turner KL. Precise frequency estimation in a microelectromechanical parametric resonator. Applied Physics Letters 2007;90(17):173508.

[28] Rhoads JF, Kumar V, Shaw SW, Turner KL. The non-linear dynamics of electromagnetically actuated microbeam resonators with purely parametric excitations. International Journal of Non-Linear Mechanics 2013;55:79-89. 
[29] Beran PS, Strganac TW, Kim K, Nichkawde C. Studies of store-induced limit-cycle oscillations using a model with full system nonlinearities. Nonlinear Dynamics 2004;37(4):323-39.

[30] Avramov KV, Pierre C, Shyriaieva NV. Nonlinear equations of flexuralflexural-torsional oscillations of rotating beams with arbitrary crosssection. International Applied Mechanics 2008;44(5):582-9.

[31] Crespo da Silva MRM, Glynn CC. Nonlinear flexural-flexural-torsional dynamics of inextensional beams. I: Equations of motion. Journal of Structural Mechanics 1978;6(4):437-48.

[32] Crespo da Silva MRM, Glynn CC. Nonlinear flexural-flexural-torsional dynamics of inextensional beams. II: Forced motions. Journal of Structural Mechanics 1978;6(4):449-61.

[33] Nayfeh AH, Pai PF. Linear and nonlinear structural mechanics. John Wiley \& Sons; 2008.

[34] Mahmoodi SN, Khadem SE, Jalili N. Theoretical development and closed-form solution of nonlinear vibrations of a directly excited nanotube-reinforced composite cantilevered beam. Archive of Applied Mechanics 2006;75(2-3):153-63.

[35] Fung YC. Foundations of Solid Mechanics. Prentice-Hall; 1965.

[36] Jeong B, Cho H, Yu MF, Vakakis AF, McFarland DM, Bergman LA. Modeling and measurement of geometrically nonlinear damping in a microcantilever nanotube system. ACS Nano 2013;7(10):8547-53.

[37] Leissa A. Vibration of Plates. National Aeronautics and Space Administration; 1969.

[38] Leissa A. The free vibration of rectangular plates. Journal of Sound and Vibration 1973;31(3):257-93.

[39] Yasumura KY, Stowe TD, Chow EM, Pfafman T, Kenny TW, Stipe BC, et al. Quality factors in micron- and submicron-thick cantilevers. Journal of Microelectromechanical Systems 2000;9(1):117-25. 
[40] Yang J, Ono T, Esashi M. Energy dissipation in submicron thick singlecrystal silicon cantilevers. Journal of Microelectromechanical Systems 2002;11(6):775-83.

[41] Eichler A, Moser J, Chaste J, Zdrojek M, Wilson-Rae I, Bachtold A. Nonlinear damping in mechanical resonators made from carbon nanotubes and graphene. Nature Nanotechnology 2011;6(6):339-42.

[42] Zaitsev S, Shtempluck O, Buks E, Gottlieb O. Nonlinear damping in a micromechanical oscillator. Nonlinear Dynamics 2012;67(1):859-83.

[43] Nayfeh AH, Mook DT. Nonlinear Oscillations. Wiley-Interscience; 1995.

[44] Rahman Z, Burton T. On higher order methods of multiple scales in non-linear oscillations-periodic steady state response. Journal of Sound and Vibration 1989;133(3):369-79.

[45] Blair KB, Krousgrill CM, Farris TN. Harmonic balance and continuation techniques in the dynamic analysis of Duffing's equation. Journal of Sound and Vibration 1997;202(5):717-31.

[46] Urabe M. Galerkin's procedure for nonlinear periodic systems. Archive for Rational Mechanics and Analysis 1965;20(2):120-52.

[47] Urabe M, Reiter A. Numerical computation of nonlinear forced oscillations by Galerkin's procedure. Journal of Mathematical Analysis and Applications 1966;14(1):107-40.

[48] Stokes A. On the approximation of nonlinear oscillations. Journal of Differential Equations 1972;12(3):535-58.

[49] Lazarus A, Thomas O. A harmonic-based method for computing the stability of periodic solutions of dynamical systems. Comptes Rendus Mecanique 2010;338(9):510-7.

[50] Sanders JA, Verhulst F. Averaging Methods in Nonlinear Dynamical Systems. New York: Springer-Verlag; 1985.

[51] Younis MI, Nayfeh AH. A study of the nonlinear response of a resonant microbeam to an electric actuation. Nonlinear Dynamics 2003;31(1):91117. 
[52] van Beek JTM, Verheijden GJA, Koops GEJ, Phan KL, van der Avoort $\mathrm{C}$, van Wingerden J, et al. Scalable 1.1 GHz fundamental mode piezoresistive silicon MEMS resonator. In: Proceedings of IEDM 2007: The IEEE Electron Devices Meeting. 2007, p. 411-4.

[53] Seber GAF, Wild CJ. Nonlinear Regression. John Wiley \& Sons; 1989.

[54] Gu M, Eisenstat S. Efficient algorithms for computing a strong rankrevealing QR factorization. SIAM Journal on Scientific Computing 1996;17(4):848-69.

[55] Pope SR. Parameter identification in lumped compartment cardiorespiratory models. Ph.D.; North Carolina State University; 2009.

[56] Donaldson JR, Schnabel RB. Computational experience with confidence regions and confidence intervals for nonlinear least squares. Technometrics 1987;29(1):67-82.

[57] Jordan DW, Smith P. Nonlinear Ordinary Differential Equations. Oxford: Oxford University Press; 2007. 
Table 5: The nominal parameters, the estimated parameters of the $N=3$ harmonic balance solution with the third-order, reduced-order model, and the estimated parameters of the $N=3$ harmonic balance solution with the fifth-order, reduced-order model for the numerical experiment when all three cases were considered simultaneously.

\begin{tabular}{|c|c|c|c|}
\hline & Nominal & $\begin{array}{c}3^{\text {rd }} \text {-Order ROM } \\
N=3 \mathrm{HB}\end{array}$ & $\begin{array}{c}5^{\text {th }} \text {-Order ROM } \\
N=3 \mathrm{HB}\end{array}$ \\
\hline$Q$ & $2.12245 \times 10^{-1}$ & $\begin{array}{c}2.12178 \times 10^{-1} \\
\pm 1.91566 \times 10^{-4}\end{array}$ & $\begin{array}{c}2.12272 \times 10^{-1} \\
\pm 2.75585 \times 10^{-4}\end{array}$ \\
\hline$k_{3}$ & 3.27127 & $\begin{array}{c}5.26443 \\
\pm 5.83821 \times 10^{-2}\end{array}$ & $\begin{array}{c}3.12479 \\
\pm 6.76691 \times 10^{-1}\end{array}$ \\
\hline$\beta$ & 4.59677 & $\begin{array}{c}7.58631 \\
\pm 8.77640 \times 10^{-2}\end{array}$ & $\begin{aligned} & 4.37696 \\
\pm & 1.01466\end{aligned}$ \\
\hline$\gamma_{3}$ & $7.54910 \times 10^{-4}$ & $\begin{array}{c}9.57825 \times 10^{-4} \\
\pm 1.78275 \times 10^{-4}\end{array}$ & $\begin{array}{c}8.93007 \times 10^{-4} \\
\pm 5.84176 \times 10^{-4}\end{array}$ \\
\hline$k_{5}$ & $1.73240 \times 10^{1}$ & NA & $\begin{array}{c}-1.58056 \\
\pm 2.11595 \times 10^{2}\end{array}$ \\
\hline$\nu_{1}$ & $5.12607 \times 10^{1}$ & NA & $\begin{array}{c}7.61167 \times 10^{1} \\
\pm 7.92745 \times 10^{3}\end{array}$ \\
\hline$\nu_{2}$ & $2.56304 \times 10^{1}$ & $\mathrm{NA}$ & $\begin{array}{c}1.48692 \times 10^{2} \\
\pm 1.39284 \times 10^{3}\end{array}$ \\
\hline$\gamma_{5}$ & $7.10725 \times 10^{-3}$ & NA & $\begin{array}{l}-5.12991 \times 10^{-2} \\
\pm 2.55375 \times 10^{-1}\end{array}$ \\
\hline$\eta_{1}$ & $6.12500 \times 10^{4} \mathrm{~A}^{-1}$ & $\begin{array}{c}6.13135 \times 10^{4} \mathrm{~A}^{-1} \\
\pm 1.74850 \times 10^{1} \mathrm{~A}^{-1}\end{array}$ & $\begin{array}{c}6.12506 \times 10^{4} \mathrm{~A}^{-1} \\
\pm 2.52925 \times 10^{1} \mathrm{~A}^{-1}\end{array}$ \\
\hline$S(\hat{\mathbf{p}})$ & $\mathrm{NA}$ & $1.37232 \times 10^{-2}$ & $1.21992 \times 10^{-2}$ \\
\hline$\hat{\sigma}_{m}$ & NA & $5.51620 \times 10^{-3}$ & $5.22410 \times 10^{-3}$ \\
\hline
\end{tabular}


Table B.6: Definitions of nondimensional parameters used in Eqn. (19).

$$
\begin{aligned}
1 & =\int_{0}^{1} \Psi^{2} \mathrm{~d} \hat{s} \\
\omega_{0}^{2} & =\int_{0}^{1} \Psi \Psi^{i v} \mathrm{~d} \hat{s} \\
\tau & =\omega_{0} \hat{t} \\
(\bullet)^{\prime} & =\frac{\partial(\bullet)}{\partial \tau} \\
k_{3} & =\frac{1}{\omega_{0}^{2}}\left(4 \int_{0}^{1} \Psi \Psi^{\prime} \Psi^{\prime \prime} \Psi^{\prime \prime \prime} \mathrm{d} \hat{s}+\int_{0}^{1} \Psi \Psi^{\prime \prime 3} \mathrm{~d} \hat{s}+\int_{0}^{1} \Psi \Psi^{\prime 2} \Psi^{i v} \mathrm{~d} \hat{s}\right) \\
k_{5} & =\frac{1}{\omega_{0}^{2}}\left(6 \int_{0}^{1} \Psi \Psi^{\prime 2} \Psi^{\prime \prime 3} \mathrm{~d} \hat{s}+8 \int_{0}^{1} \Psi \Psi^{\prime 3} \Psi^{\prime \prime} \Psi^{\prime \prime \prime} \mathrm{d} \hat{s}+\int_{0}^{1} \Psi \Psi^{\prime 4} \Psi^{i v} \mathrm{~d} \hat{s}\right) \\
\beta & =\int_{0}^{1} \Psi \Psi^{\prime \prime} \int_{1}^{\hat{s}} \int_{0}^{\hat{s}_{2}} \Psi^{\prime 2} \mathrm{~d} \hat{s}_{1} \mathrm{~d} \hat{s}_{2} \mathrm{~d} \hat{s}+\int_{0}^{1} \Psi \Psi^{\prime} \int_{1}^{\hat{s}} \int_{0}^{\hat{s}_{2}} \Psi^{\prime 2} \mathrm{~d} \hat{s}_{1} \mathrm{~d} \hat{s} \\
\nu_{1} & =\frac{3}{2} \int_{0}^{1} \Psi \Psi^{\prime \prime} \int_{1}^{\hat{s}_{2}} \int_{0}^{\prime 4} \Psi^{\prime 4} \mathrm{~d} \hat{s}_{1} \mathrm{~d} \hat{s}_{2} \mathrm{~d} \hat{s}+\frac{3}{2} \int_{0}^{1} \Psi \Psi^{\prime} \int_{0}^{\hat{s}_{2}} \Psi^{\prime 4} \mathrm{~d} \hat{s}_{1} \mathrm{~d} \hat{s} \\
& +\frac{3}{2} \int_{0}^{1} \Psi \Psi^{\prime 2} \Psi^{\prime \prime} \int_{1}^{\hat{s}} \int_{0}^{\hat{s}_{2}} \Psi^{\prime 2} \mathrm{~d} \hat{s}_{1} \mathrm{~d} \hat{s}_{2} \mathrm{~d} \hat{s}+\frac{1}{2} \int_{0}^{1} \Psi \Psi^{\prime 3} \int_{0}^{\hat{s}} \Psi^{\prime 2} \mathrm{~d} \hat{s}_{1} \mathrm{~d} \hat{s}
\end{aligned}
$$


Table B.7: Definitions of nondimensional parameters used in Eqn. (19).

$$
\begin{aligned}
\nu_{2} & =\frac{1}{2} \int_{0}^{1} \Psi \Psi^{\prime \prime} \int_{1}^{\hat{s}} \int_{0}^{\hat{s}_{2}} \Psi^{\prime 4} \mathrm{~d} \hat{s}_{1} \mathrm{~d} \hat{s}_{2} \mathrm{~d} \hat{s}+\frac{1}{2} \int_{0}^{1} \Psi \Psi^{\prime} \int_{0}^{\hat{s}} \Psi^{\prime 4} \mathrm{~d} \hat{s}_{1} \mathrm{~d} \hat{s} \\
& +\frac{3}{2} \int_{0}^{1} \Psi \Psi^{\prime 2} \Psi^{\prime \prime} \int_{1}^{\hat{s}} \int_{0}^{\hat{s}_{2}} \Psi^{\prime 2} \mathrm{~d} \hat{s}_{1} \mathrm{~d} \hat{s}_{2} \mathrm{~d} \hat{s}+\frac{1}{2} \int_{0}^{1} \Psi \Psi^{\prime 3} \int_{0}^{\hat{s}} \Psi^{\prime 2} \mathrm{~d} \hat{s}_{1} \mathrm{~d} \hat{s} \\
\lambda_{1} & =\frac{g B_{y} l^{4}}{E I v_{0}^{2} \omega_{0}^{2}} \int_{0}^{1} \Psi^{\prime 2} \mathrm{~d} \hat{s} \\
\lambda_{3} & =\frac{g B_{y} l^{4}}{2 E I v_{0}^{2} \omega_{0}^{2}} \int_{0}^{1} \Psi^{\prime 4} \mathrm{~d} \hat{s} \\
\lambda_{5} & =\frac{3 g B_{y} l^{4}}{8 E I v_{0}^{2} \omega_{0}^{2}} \int_{0}^{1} \Psi^{\prime 6} \mathrm{~d} \hat{s} \\
\eta_{1} & =-\left.\frac{g B_{x} l^{5}}{E I v_{0}^{3} \omega_{0}^{2}} \Psi\right|_{\hat{s}=1} \\
Q & =\frac{E T v_{0}^{2}}{G \omega_{0} l^{2}} \\
\gamma_{3} & =\frac{G}{E T \omega_{0}}\left(-6 \int_{0}^{1} \Psi \Psi^{\prime} \Psi^{\prime \prime} \Psi^{\prime \prime \prime} \mathrm{d} \hat{s}-3 \int_{0}^{1} \Psi \Psi^{\prime \prime 3} \mathrm{~d} \hat{s}\right) \\
\gamma_{5} & =\frac{G}{E T \omega_{0}}\left(-9 \int_{0}^{1} \Psi \Psi^{\prime 2} \Psi^{\prime \prime 3} \mathrm{~d} \hat{s}-3 \int_{0}^{1} \Psi \Psi^{\prime 3} \Psi^{\prime \prime} \Psi^{\prime \prime \prime} \mathrm{d} \hat{s}+\int_{0}^{1} \Psi \Psi^{\prime 4} \Psi^{i v} \mathrm{~d} \hat{s}\right)
\end{aligned}
$$

